\title{
Oral, but not rectal delivery of epigallocatechin-3- gallate alleviates colitis by regulating the gut microbiota, oxidative stress, inflammation, and barrier integrity
}

\section{Zhenhua Wu}

China Agricultural University

Shimeng Huang

China Agricultural University

Tiantian Li

China Agricultural University

$\mathrm{Na} \mathrm{Li}$

China Agricultural University

Dandan Han

China Agricultural University

Shiyi Zhang

China Agricultural University

Jiaman Pang

China Agricultural University

Shilan Wang

China Agricultural University

Guolong Zhang

Oklahoma State University

Junjun Wang ( $\nabla$ wangjj@cau.edu.cn )

China Agricultural University https://orcid.org/0000-0001-9427-3824

Research

Keywords: Colitis, EGCG, microbiota, SCFAs, oxidative stress, mucosal barrier function

Posted Date: August 6th, 2020

DOl: https://doi.org/10.21203/rs.3.rs-52274/v1

License: (9) This work is licensed under a Creative Commons Attribution 4.0 International License. Read Full License 
Page $2 / 33$ 


\section{Abstract}

Background: Alteration of the gut microbiota may contribute to the development of inflammatory bowel diseases (IBDs). Epigallocatechin-3-gallate (EGCG), a major bioactive constituent of green tea, is known to be beneficial in IBDs alleviation. However, it is unclear whether EGCG attenuates IBDs through direct improvement of gastrointestinal function or indirect alteration of the structure and function of the gut microbiota.

Results: We first investigated the therapeutic effects of EGCG on disease severity, oxidative stress, inflammation, barrier function, and gut microbiota in murine colitis model, and further demonstrate it via EGCG pre-supplementation. We revealed that, oral, but not rectal, delivery of EGCG alleviated the severity of colitis through attenuation of anti-oxidative and anti-inflammatory response. Mucin-secreting goblet cell number, barrier function gene expression levels, and the integrity of tight junctions in the colon were also enhanced by oral EGCG. Additionally, we observed distinct EGCG-mediated alternation in the gut microbiome, as highlighted by increased Akkermansia abundance and butyrate production. Furthermore, we revealed that prophylactic oral application of EGCG for 21 days prior to the onset of dextran sodium sulfate (DSS)-induced colitis also ameliorated colonic damage, oxidative stress, and inflammatory response. Prophylactic EGCG significantly enriched Akkermansia, Faecalibaculum, and Bifidobacterium and enhanced acetate, propionate and butyrate production in DSS-treated mice. Moreover, scores of differential microbes, in particular Akkermansia, showed a strong positive correlation with short-chain fatty acids (SCFAs) and antioxidant enzyme levels in both the plasma and colon, but a negative association with inflammatory cytokines and malondialdehyde.

Conclusions: EGCG is capable of treating DSS-induced colitis both therapeutically and prophylactically by inducing a pronounced anti-oxidative and anti-inflammatory response. Attenuation of colitis by oral, but not rectal administration of, EGCG suggests an intimate involvement of the gut microbiota. Increased Akkermansia and subsequent protective SCFAs production may be largely responsible for the antiinflammatory and anti-oxidative function of EGCG, leading to restoration of intestinal epithelial homeostasis of the host. These findings provide novel insights into EGCG-mediated remission of IBDs and the rationale for devising more effective therapeutic strategies for IBDs.

\section{Introduction}

Inflammatory bowel diseases (IBDs), clinically consisting of ulcerative colitis (UC) and Crohn's disease (CD), are chronic inflammatory diseases of the gastrointestinal tract (GIT) [1]. They are becoming more prevalent worldwide in recent years [2]. The facts that IBDs are associated with dysregulation of the gut microbiome $[3,4]$ and that dietary preferences could alter the risk to IBDs suggest possible involvement of the gut microbiota in IBDs development $[5,6]$. Therefore, manipulation of the gut microbiome has potential for IBDs therapy $[7,8]$. 
Daily consumption of teas has been linked to a reduced risk to IBDs $[5,9,10]$. Tea is known to exert a prebiotic-like effect and modulate the gut microbiota [11]. Conversely, the gut microbiota also contributes to the metabolism of tea polyphenols to facilitate the clearance of toxic reactive metabolites in cells [12], suggesting the cross-talk between gut microbiota and host oxidative and inflammatory response is pivotal to maintain intestinal health and barrier function [13-16].

Epigallocatechin-3-gallate (EGCG), a major bioactive polyphenol in green tea, possesses antiinflammatory and anti-oxidative properties both in vitro and in vivo $[17,18]$, with known therapeutic benefits in a murine model of colitis $[19,20]$. Considering the poor bioavailability of EGCG in vivo [21], it is unclear whether EGCG-mediated alleviation of IBDs acts through modulation of the inflammatory and oxidative status of the GIT or the gut microbiome.

Herein, we hypothesized that attenuation of colitis by EGCG is mediated mainly through modulation in the gut microbiome, which subsequently leads to alleviation of inflammation and oxidative stress in the host $[6,22]$. The effect of oral or rectal administration of EGCG was tested in a murine model of DSSinduced colitis. The oxidative and inflammatory response of the host, and the alteration of the intestinal bacteria and production of SCFAs were further investigated. Our results suggest that an obvious shift in the gut microbiota and subsequent increased production of SCFAs are largely responsible for the in vivo anti-oxidative and anti-inflammatory effects of EGCG, which collectively contribute to the resolution of intestinal inflammation and oxidative damage in IBDs.

\section{Results}

\section{Oral, but not rectal, administration of EGCG alleviates DSS-induced colitis}

To study the impact of EGCG on alleviation of DSS-induced colitis, an experimental colitis was induced in mice by administering $2.5 \%$ DSS in water continuously for seven days, followed by three days of daily oral (Fig. 1A) or rectal delivery (Fig. S1A) of $50 \mathrm{mg} / \mathrm{kg}$ body weight EGCG, as the optimal dose we have explored before (data not shown). To explore how oral EGCG or enteric delivery EGCG attenuates DSSinduced colitis, the symptoms of mice treated by two different exposure ways were measured. Oral administration of EGCG significantly alleviated colitis as measured by disease activity index (DAl, the combined score of body weight loss, stool consistency, and rectal bleeding [23]) (Fig. 1B), body weight loss (Fig. 1C), and colon length (Fig. 1D and 1E). However, rectal delivery of EGCG failed to improve any of the measurements above (Fig. S1B, S1C, S1D, and S1E). Histological analysis further showed obvious attenuation of inflammatory cell infiltration and mucosal damage and overall histology score in the colon in response to oral (Fig. 1F and 1G), but not rectal, administration of EGCG (Fig. S1F and S1G). Remarkably, during the whole experiment period, one mouse from DSS + Oral-PBS group was found dead on the tenth day, and one mouse from DSS + Rectal-PBS group and DSS + Rectal-EGCG group respectively were found dead on the ninth day.

To further assess the impact of EGCG on systemic and intestinal inflammatory response, myeloperoxidase (MPO) and pro-inflammatory cytokines in the plasma and colonic tissues were 
measured. Plasma concentrations of IL-1 $\beta$ (Fig. 1H), IL-6 (Fig. 1I), IL-8 (Fig. 1J), and TNF-a (Fig. 1K) were significantly decreased in DSS-treated mice in response to oral EGCG. In contrast, rectal EGCG failed to suppress the levels of any cytokine in the plasma (Fig. S1H, S1I, S1J, and S1K). MPO production is often increased in the colon of IBDs patients [24]. Similarly, DSS drastically elevated the MPO concentration in the colon of mice, but such an elevation was significantly dampened by oral EGCG (Fig. 1L). Furthermore, both oral and rectal EGCG significantly decreased the IL-6 level in the colon (Fig. 1M and S1M) with TNFa showing a strong tendency to decrease as well (Fig. $1 \mathbf{N}$ and S1N). However, rectal administration of EGCG failed to suppress the levels of MPO (Fig. S1L) in the colon of DSS-treated mice.

Collectively, these results indicated that clinical colitis symptoms and colonic damage were ameliorated by oral, but not rectal administration of EGCG.

\section{Oral administration of EGCG suppresses DSS-induced oxidative stress of the intestinal mucosa and restores barrier function}

To assess the influence of EGCG on oxidative stress, total antioxidant capacity (T-AOC), total superoxide dismutases (T-SOD), catalase (CAT), glutathione peroxidase (GSH-Px), and malondialdehyde (MDA) in the plasma and colon were measured. DSS significantly suppressed the T-SOD and CAT concentrations in the plasma, which, however, were largely restored by oral administration of EGCG (Fig. 2A and 2B). On the other hand, the MDA level in the plasma was significantly increased by DSS, but returned to normal by EGCG (Fig. 2C). Moreover, increased MDA in the plasma was also returned to normal by rectal EGCG (Fig. S2C). However, DSS-mediated suppression of the T-SOD and CAT concentrations in the plasma were not obviously impacted by rectal EGCG (Fig. S2). In the colon, T-AOC and T-SOD were significantly decreased by DSS, but restored to normal by oral EGCG (Fig. 2D and 2E). No significant differences in the levels of CAT (Fig. 2F) or GSH-px (Fig. 2G) were observed in the colon in response to DSS with or without oral EGCG. Colonic production of MDA was significantly increased by DSS, but reduced to normal by EGCG (Fig. 2H). However, the effect of DSS on T-AOC (Fig. S2D), T-SOD (Fig. S2E) or MDA (Fig. S2H) was not obviously impacted by rectal EGCG. Of note, the level of GSH-px in the colon was significantly increased by rectal EGCG even compared with the colon of the normal group (Fig. S2G). Furthermore, TUNEL assay revealed that DSS caused a significant increase in apoptotic in the colonic mucosa; however, both oral and rectal EGCG had no obvious beneficial effect on reducing apoptosis (Fig. 2l, 2K, S2I and S2K). To assess the effect of oral or rectal EGCG on the colonic mucosal barrier function, we counted mucinsecreting goblet cells in the colonic epithelia using Alcian blue staining. DSS significantly reduced the frequency of goblet cells and the thickness of colonic epithelial mucosa, and desirably, oral EGCG restored such damping to normal (Fig. 2J and 2L). Consistently, oral EGCG largely reversed DSS disruption of the fine structure of the brush border and tight junctions as revealed by transmission electron microscopy (TEM) (Fig. 2M). However, no obvious difference in the frequency of goblet cells or the thickness of colonic epithelial mucosa and fine structure was observed in response to DSS with or without rectal EGCG (Fig. S2J, S2L and S2M). 
These results suggested that oral, but not rectal, EGCG suppressed DSS-induced oxidative stress, regulated the cell apoptosis of colonic epithelium, and restored mucosal barrier function.

\section{Oral administration of EGCG alters the gut microbiota composition and metabolism}

To further explore the impact of EGCG on the gut microbiota composition, 16S rDNA gene sequencing was performed with the fecal samples of DSS-treated mice receiving oral or rectal administration of EGCG. Neither oral (Fig. 3A) nor rectal EGCG (Fig. S3A) had a significant influence on richness of the fecal microbiota as indicated by the Sobs index. However, principal co-ordinates analysis (PCoA) showed a clear separation in the gut microbiota of DSS-treated mice between the DSS + Oral-EGCG group and DSS + Oral-PBS group $(R=0.1684, P=0.0360)$ (Fig. 3B), indicating that the gut microbiota structure was significantly impacted by oral EGCG. The fecal microbiota structure was also partly influenced by rectal $\operatorname{EGCG}(R=0.2177, P=0.0110)$ (Fig. S3B).

To identify differentially enriched fecal bacterial taxa in DSS-treated mice in response to oral or rectal administration of EGCG, we explored the overall fecal bacterial composition of mice at the phylum and genus level by barplot and further performed differentia bacteria by LEfSe analysis. At the phylum level, Bacteroidetes, Firmicutes, and Proteobacteria were predominant phyla in the fecal microbiota (Fig. 3C and Fig. S3C). At the genus level, the fecal microbiota was dominated by Bacteroids, Lactobacillus, Escherichia-Shigella (Fig. 3D and Fig. S3D). Additionally, we found that two members of Akkermansia and UBA1819 were enriched by oral EGCG, while fourteen other taxa were enriched in the DSS only group (Fig. 3E). Seven bacterial taxa such as two members of Alloprevotella and Actinobacillus were particularly abundant in response to rectal EGCG, while eight other taxa such as members of Lactobacillus, Turicibacter, and Romboutsia were enriched in the DSS only group (Fig. S3E). Moreover, to assess how oral and rectal EGCG impacted the function of gut microbiota, we performed PICRUSt and revealed that multiple KEGG pathways such as metabolism of amino acids, energy, and lipids as well as membrane transport were enriched in response to oral EGCG administration (Fig. 3F and S3F).

To further explore the effect of oral or rectal EGCG on the synthesis of SCFAs, we measured the fecal concentrations of acetate, propionate, and butyrate. All three SCFAs were significantly diminished by DSS, but the productions of propionate and butyrate were significantly restored by oral (Fig. $2 \mathrm{H}$ and $\mathbf{2 l}$ ), but not rectal (Fig. S2H and S2I), administration of EGCG. In fact, butyrate production was further significantly reduced in DSS-treated mice in response to rectal EGCG (Fig. S2I).

Alteration of the gut microbiota and increased production of SCFAs occurred in response to oral, but not rectal EGCG administration suggested that gut microbiota might play a critical role in alleviating DSSinduced colitis.

Correlation between the gut microbiota and anti-oxidative and inflammatory parameters and SCFAs profiles 
Spearman correlation analysis was further performed to understand the association between differentially enriched microbes and anti-oxidative, inflammatory parameters or SCFAs profiles. Correlation heatmap revealed that Akkermansia had a strong positive correlation $(P<0.05)$ with the TAOC level in the colon and the butyrate level in the feces, but a significant negative correlation $(P<0.05)$ with IL-6 in the colon and IL-8 in the plasma (Fig. 4). Meanwhile, it revealed that UBA1819 showed a significantly positive correlation $(P<0.05)$ with the levels of T-SOD and CAT in the plasma and the production of propionate and butyrate. A significantly negative correlation $(P<0.05)$ between UBA1819 and the levels of IL-1 $\beta$ and IL- 6 in the plasma and the levels of IL- 6 and MDA in the colon was also observed (Fig. 4).

Above all, a strong correlation between the differentia microbiotas such as Akkermansia and UBA1819 strongly and the anti-oxidative and inflammatory parameters and SCFAs profiles might suggest a critical involvement of these bacteria in mediating EGCG alleviation of colitis.

\section{Prophylactic EGCG alleviates DSS-induced colitis}

Given the benefit of green tea consumption in reducing the risk of IBDs [10] and different impact on the gut microbiota by oral and rectal delivery of EGCG, we hypothesized that EGCG could alleviate DSSinduced colitis prophylactically. Mice were subjected to DSS in drinking water for five days to induce acute colitis after receiving daily oral gavage of $50 \mathrm{mg} / \mathrm{kg}$ body weight EGCG for 21 days (Fig. 5A), also as a simulation of about four to eight cups of tea per day for an adult [25]. Disease symptoms and body weight were monitored daily. Mice were then sacrificed and the colonic pathology was evaluated. The concentrations of several representative inflammatory mediators were also measured in both the plasma and colon. Apparently, prophylactic EGCG alleviated the symptoms of colitis in DSS-treated mice as indicated by significantly reduced daily DAI (Fig. 5B) and body weight loss (Fig. 5C), and increased length of colon (Fig. 5D and 5E). Histological analysis further revealed that prophylactic EGCG suppressed DSSinduced infiltration of inflammatory cells and damage to the colonic mucosa (Fig. $\mathbf{5 F}$ and $\mathbf{5 G}$ ). Moreover, EGCG pre-supplementation tended to reduce the plasma levels of IL-1 $\beta$ (Fig. 5H), IL-6 (Fig. 5I), IL-8 (Fig. 5J), and TNF-a (Fig. 5K) in DSS mice. Strikingly, DSS-induced elevations of MPO (Fig. 5L), IL-6 (Fig. 5M), and TNF-a (Fig. $\mathbf{5 N}$ ) in the colon were largely restored to normal levels by prophylactic EGCG. It is noted that the same dose of EGCG pre-supplementation caused essentially no alterations of any of the aforementioned parameters to normal mice, except for a significant reduction in the colonic levels of three mediators (MPO, IL-6, and TNF-a) (Fig. 5L, 5M, and 5N).

These results collectively indicated that prophylactic EGCG is capable of suppressing DSS-induced colitis symptoms, colonic injury, and inflammation.

\section{Prophylactic EGCG reduces DSS-induced oxidative stress and apoptosis and improves the mucosal barrier function}

Prophylactic EGCG largely restored the plasma levels of antioxidant enzymes, T-SOD and CAT that were significantly reduced by DSS (Fig. 6A and 6B). Consistently, MDA in the plasma was significantly 
increased by DSS, but reduced to normal in response to prophylactic EGCG (Fig. 6C). Similarly, the colonic levels of T-AOC (Fig. 6D), T-SOD (Fig. 6E), CAT (Fig. 6F), and GSH-px (Fig. 6G) were significantly reduced by DSS, but were largely restored to the normal levels by prophylactic EGCG. The colonic level of MDA was significantly increased by DSS, but returned below normal in response to prophylactic EGCG (Fig. $6 \mathrm{H})$. Of note, EGCG pre-supplementation even elicited an obvious anti-oxidative response in both the plasma and colon in healthy mice (Fig. 6A-6H).

TUNEL analysis further revealed that DSS significantly increased the frequency of apoptotic cells in the colonic mucosa, but prophylactic EGCG largely reversed the trend (Fig. $\mathbf{6 I}$ and $6 \mathrm{~K}$ ). Moreover, prophylactic EGCG also restored the number of Alcian blue-positive goblet cells in the colon that were reduced by DSS (Fig. 6J and 6L). TEM revealed prophylactic EGCG-mediated preservation of the brush border and tight junctions of the colonic mucosa that were damaged by DSS (Fig. 6M). Consistently, the mRNA expression levels of several major barrier function genes such as Claudin-1 (Fig. 6A), Occludin (Fig. 6B), Zona occludens 1 (ZO-1) (Fig. 7C), Mucin-1 (Fig. 7D), and Mucin-2 (Fig. 7E), were suppressed by DSS, but restored to or raised above their normal levels (as in the case of Claudin-1, Mucin-1 and Mucin-2) in response to prophylactic EGCG.

These results suggested that prophylactic EGCG is capable of suppressing DSS-triggered oxidative stress, regulating apoptosis and restoring mucosal barrier function in colonic epithelia.

\section{Prophylactic EGCG shifts the gut microbiota composition and metabolism}

Next, we investigated the impact of prophylactic EGCG on the gut microbiota composition of DSS-treated mice. Sobs Index was not affected by prophylactic EGCG in healthy or DSS-treated mice, although DSS caused a significant reduction $(P<0.001)$ in richness of the gut microbiota (Fig. 8A). PCoA analysis showed a clear separation between groups with and without DSS $(R=0.5160, P=0.001$, Fig. 8B). Additionally, prophylactic EGCG significantly shifted the gut microbiota composition in both healthy ( $R=$ 0.4277, $P=0.001$, Fig. $8 \mathrm{C})$ and DSS-treated mice $(R=0.6138, P=0.001$, Fig. 8D). Meanwhile, we explored the overall fecal bacterial composition of mice at the phylum and genus level by barplot. At the phylum level, Bacteroidetes, Firmicutes, and Proteobacteria were predominant in these groups (Fig. 8E). At the genus level, fecal microbiota was dominated by Bacteroids, Lachnospiraceae_NK4A136_group, Odoribacter and Lactobacillus (Fig. 8F). Indeed, LEfSe analysis revealed differential enrichment of fecal bacteria in DSS-treated mice in response to prophylactic EGCG (Fig. 8G). While ten bacterial genera such as Akkermansia, Faecalibaculum, and Bifidobacterium were enriched by EGCG, another ten genera such as Bacteroides were enriched in the DSS only group. Furthermore, PICRUSt analysis predicted an enrichment of multiple pathways such as amino acid metabolism, energy metabolism, and translation in DSS-treated mice in response to prophylactic EGCG (Fig. $\mathbf{8 H}$ ). It is noteworthy that oral administration of EGCG to healthy mice caused an enrichment of 17 bacterial genera such as such as Akkermansia and UBA1819 (Fig. S4), which are associated with increased metabolism in carbohydrates and lipids and membrane transport (Fig. S5). Additionally, prophylactic EGCG completely restored the production of SCFAs such as acetate (Fig. 8I), propionate (Fig. 8J), and butyrate (Fig. 8K) in DSS-treated mice. 


\section{Correlation between differentially microbes induced by prophylactic EGCG and anti-oxidative, inflammatory parameters, and SCFAs profiles}

Spearman correlation analysis of DSS-treated mice with and without receiving prophylactic EGCG revealed that 20 different fecal bacterial genera showed a significant correlation $(P<0.05)$ with the concentrations of at least one anti-oxidative or pro-inflammatory mediator in the plasma or SCFAs in the feces. For example, Akkermansia, Bifidobacterium and Faecalibaculum were positively correlated $(P<$ 0.05 ) with CAT level in the plasma, T-AOC, T-SOD, CAT, GSH-px levels in the colon, and acetate, propionate, and butyrate in the feces, but with a negative correlation $(P<0.05)$ with the colonic levels of MDA, IL-6, TNF-a, and MPO (Fig. 9). Akkermansia and Faecalibaculum also showed a significantly positive correlation $(P<0.05)$ with T-SOD in plasma, but a negative correlation $(P<0.05)$ with the MDA level in plasma (Fig. 9). Additionally, a number of bacteria such as Akkermansia showed a significant correlation $(P<0.05)$ with the concentrations of anti-oxidative and inflammatory mediators as well as fecal SCFAs in healthy mice with or without receiving EGCG (Fig. S5).

Above, these results indicated that prophylactic EGCG regulates the gut microbiota composition and its metabolism, leading to a potential to restore the DSS-induced dysbiosis.

\section{Discussion}

Besides host genetics, such as dietary preference is associated with risks to IBDs [6, 10,26,27]. Daily consumption of tea has been shown to reduce the risk of IBDs $[9,10]$. As a major bioactive component of green tea, EGCG possesses strong anti-oxidative and anti-inflammatory properties [19, 20, 28-32], and its protective effect results from the modulation on gut microbiome arises the attention [33]. In fact, gut microbiota constitutes a critical bridge between environmental factors and host health [34-36], and IBDs are associated with dramatic changes in the gut microbiome [3]. Although it has been shown to alleviate the severity of DSS-induced colitis in mice $[19,20]$, whether EGCG works through modulation of the gut microbiota remains elusive.

In this study, we confirmed that therapeutic applications of oral EGCG has led to alleviation of DSSinduced colitis symptoms and histological damages, which was in agreement with previous studies [19, 20]. In addition, we demonstrated that benefits of prophylactic EGCG in alleviation of DSS-induced colitis. Consistent with findings from previous studies [19, 37-39], oral administration of EGCG also suppressed colonic inflammation as exemplified by suppression of inflammatory cytokines such as IL-6 and TNF-a in both the plasma and colon of DSS-treated mice. Furthermore, we found that rectal delivery of EGCG failed to attenuate intestinal inflammation and pathology, which was also supported by its little toxicity on rectal mucosa [40]. Moreover, we showed in this study that both prophylactic and therapeutic oral administration of EGCG significantly attenuated DSS-induced oxidative stress by increasing the synthesis of antioxidant enzymes such as T-SOD and CAT, and decreasing the production of MDA in the colon and plasma. In agreement with it, previous study has revealed that therapeutic EGCG shows similar antioxidative effects [20], while our results further revealed that prophylactic EGCG was also capable of 
increasing the level of GSH-px in the colon. Moreover, we demonstrated that EGCG especially prophylactic EGCG regulated apoptosis, attenuated DSS-induced damage in integrity and function of barrier as evidenced by increased goblet cells and barrier function gene expression, as well as enhanced tight junctions and ultrastructure of the colonic mucosa, which is in agreement with previous studies [41-44].

Importantly, our results indicated that oral and rectal deliveries of EGCG differentially regulated the gut microbiota and its metabolism. Of note, both therapeutic and prophylactic EGCG significantly increased Akkermansia and promoted the production of SCFAs. In accordance with previous studies, it has also shown the enrichment of Akkermansia by EGCG treatment $[45,46]$ though its richness in microbiota metabolites was unclear. Furthermore, according our present study, in response to oral but not rectal EGCG, Akkermansia correlated positively with SCFAs production, antioxidant enzymes, with a negative correlation with inflammatory cytokines. On the other hand, Akkermansia was not enriched by rectal delivery of EGCG. Consistently, Akkermansia has shown similar correlation with that from oral therapy of EGCG in response to prophylactic EGCG. Consistent with it, numerous studies have already paid attention to the interplay between gut microbiome regulated by green tea polyphenols and alleviation or preventing in diseases and obesity, and the key microbe Akkermansia plays crucial role [47-49]. As a common intestinal commensal bacterium, Akkermansia has an anti-inflammatory role [50,51]. Not surprisingly, Akkermansia muciniphila is found to be reduced in IBDs patients [52] and also inversely correlates with the severity of acute appendicitis [53], which is a different form of local intestinal inflammation. Perhaps as a piece of direct evidence, a strain of Akkermansia muciniphila (ATCC BAA-835) was recently shown to exert anti-inflammatory activities by suppressing inflammatory cytokines secretion in cell culture and further ameliorate DSS-induced colitis in mice [54]. In the same study, SCFAs production was also increased in response to oral gavage of Akkermansia muciniphila [54]. In reverse, Lactobacillus levels and butyrate production in colitis mice were further decreased by rectal administration of EGCG compared to the colitis mice. As a common bacteria, Lactobacillus is considered to exert anti-inflammatory effects [55]. And previous research has demonstrated that decreasing Lactobacillus levels and butyrate production result in exacerbation of colitis in mice [6].

In addition to Akkermansia, we also revealed that prophylactic EGCG contributed to the enrichment in several other microbes such as Faecalibaculum and Bifidobacterium and enhanced the production of acetate, propionate, and butyrate. We further demonstrated that Faecalibaculum, and Bifidobacterium also correlated positively with SCFAs production, antioxidant enzymes, correlated negatively with inflammatory cytokines. Supported by a recent study, Faecalibaculum rodentium is strongly suppressed during intestinal tumorigenesis and produces protective SCFAs to inhibit tumor cell proliferation [56]. Similarly, Bifidobacterium is another SCFAs-producing bacterium with profound immune modulatory effects [57]. It is worth mentioning that, although Akkermansia is likely a major microbe that mediates much of the EGCG benefits, several other SCFAs-producing bacteria such as Faecalibaculum and Bifidobacterium $[56,57]$ enriched in response to prophylactic EGCG are also of concern.

Collectively, our results suggested that EGCG-mediated alternations in the gut microbiome might play a key role in alleviation of colitis. The difference in the gut microbiome is likely to be the major driving force 
behind therapeutic outcomes between oral and rectal deliveries of EGCG. Considering the modulation of epithelial integrity and mucosal immunity by the microbiota and microbiota-derived metabolites [58, 59], and the correlation we have revealed here, we concluded that the gut microbiome induced by EGCG contributes to the homeostatic balance of colon. This conclusion is further supported by the fact that EGCG has a low bioavailability and is poorly absorbed by the GI tract and thus its direct impact on host physiology is believed to be rather limited [21]. Taken together, based on these results, we concluded that several SCFAs-producing bacteria, especially which were induced by prophylactic EGCG, Akkermansia, Faecalibaculum, and Bifidobacterium, and subsequent increased production of protective SCFAs contributes to the anti-oxidative and anti-inflammatory state, and further protects from the damage in colon.

\section{Conclusion}

In summary, oral, but not rectal, delivery of EGCG attenuates DSS-induced colitis and leads to characteristic enrichment of Akkermansia, increased synthesis of SCFAs, suppression of oxidative stress and inflammation, and promotion of mucosal barrier integrity in DSS-treatment mice regardless whether administered therapeutically or prophylactically. Given poor bioavailability of EGCG and a known ability of Akkermansia to produce SCFAs, elicit an anti-inflammatory response, and ameliorate DSS-induced colitis, we believe that EGCG attenuates IBDs progress mainly through an alteration of the structure and function of the intestinal microbiota and enrichment of Akkermansia in particular. These microbiota changes subsequently lead to an increased production of SCFAs such as butyrate, which in turn trigger a pronounced anti-oxidative, anti-inflammatory, and barrier-enhancing program, resulting in attenuation of intestinal inflammation and damage. These results are summarized in Fig. 10. Taken together, these findings provide novel insights into EGCG-mediated alleviation of IBDs and will facilitate the development of novel and more effective therapeutic and preventive strategies for IBDs and other inflammatory disorders.

\section{Material And Methods}

\section{Animals and sample collection}

Seven- to eight-week-old specific pathogen-free (SPF) female C57L/6J mice (SPF Biotechnology Co., Ltd, Beijing, China) were maintained with four animals per cage and housed in a standard SPF facility of China Agricultural University with a 12-h light and 12-h dark cycle at $22^{\circ} \mathrm{C}$. Colitis was induced by administration of DSS (molecular mass 36 - 40 kDa, MP Biologicals, Solon, OH, USA) through drinking water.

After one week of acclimation, mice were randomly divided into six treatments with two cages of four mice per treatment. Animals were provided with free access to tap water supplemented with or without $2.5 \%$ DSS for seven days, followed by daily oral or rectal administration of $100 \mu \mathrm{L}$ PBS in the presence or absence of $50 \mathrm{mg} / \mathrm{kg}$ body weight of EGCG (purity $\geq 98 \%$, Tokyo Chemical Industry Co., Ltd., Tokyo, 
Japan) for another three days. The treatment groups are as follows: 1) Oral-CON group: tap water for seven days, followed by daily oral administration of PBS for three days; 2) DSS + Oral-PBS group: $2.5 \%$ DSS for seven days, followed by daily oral administration of PBS for three days; 3) DSS + Oral-EGCG group: $2.5 \%$ DSS for seven days, followed by daily oral administration of EGCG for three days; 4) RectalCON group: tap water for seven days, followed by daily rectal administration of PBS for three days; 2) DSS + Rectal-PBS group: 2.5\% DSS for seven days, followed by daily rectal administration of PBS for three days; 3) DSS + Rectal-EGCG group: $2.5 \%$ DSS for seven days, followed by daily rectal administration of EGCG for three days.

Body weight was measured daily for the entire duration of the study. Disease activity index (DAI) was evaluated to assess the severity of the colitis by combining scores of body weight loss, diarrhea of the stool, and the extent of blood in the feces $[60,61]$. The mice were sacrificed after anesthesia on day 11 , the length of the colon was measured, and fecal samples of all mice were collected and stored at $-80^{\circ} \mathrm{C}$ for future analysis. Plasma was obtained by centrifugation $\left(3000 \times \mathrm{rpm}\right.$ for $15 \mathrm{~min}$ at $\left.4^{\circ} \mathrm{C}\right)$ and then stored at $-80^{\circ} \mathrm{C}$. A $5 \mathrm{~mm}$ segment of the mid-colon was flushed with PBS and then fixed in $10 \%$ formalin for subsequent histology, while the remaining colon tissue was washed with PBS and snap frozen in liquid nitrogen for future analyses.

To study the prophylactic efficacy of EGCG in alleviating colitis, another experiment was conducted using seven- to eight-week-old SPF female C57L/6J mice. After one week of acclimation, mice were randomly divided into four groups with eight mice per group and received daily oral gavage of $100 \mu \mathrm{L}$ PBS alone or the same volume of PBS containing $50 \mathrm{mg} / \mathrm{kg}$ body weight of EGCG for four weeks, with or without $2.5 \%$ DSS in drinking water for five days to induce acute colitis. The treatment groups are as follows: 1) CON group: daily gavage of PBS with regular tap water for four weeks; 2) EGCG group: daily gavage of EGCG with regular tap water for four weeks; 3) DSS group: daily gavage of PBS for four weeks with $2.5 \%$ DSS in drinking water in the last week; 4) EGCG + DSS group: daily gavage of EGCG for four weeks with $2.5 \%$ DSS in drinking water for five days. From day 22 to day 26, body weight and DAl were measured daily. All mice were sacrificed upon anesthesia on day 26 . The length of the colon was measured, and a 5-mm segment of the mid-colon was fixed in formalin for sectioning and staining. The feces and the remaining colon were sampled from each mouse and snap frozen in liquid nitrogen for future analyses. Plasma was also prepared from the blood and frozen at $-80^{\circ} \mathrm{C}$ for future measurement of the cytokine concentrations.

\section{Histological analysis}

For morphological measurements, formalin-fixed colon tissues were sectioned and stained with hematoxylin and eosin. The images were evaluated using the Image $\mathrm{J}$ software (US National Institutes of Health, Bethesda, MD). The extent of inflammatory infiltration, histopathological changes in crypt structure, ulceration and crypt loss, ulcer, and presence or absence of edema were measured, and the histological score was determined as previously described [60,61]. To count colonic goblet cells, fixed colonic tissues were also stained in Alcian blue for 10-15 min and dehydrated with $100 \%$ alcohol and 
xylene, followed by image acquisition on a microscope (Carl Zeiss AG, Jena, Germany). Acidic mucuscontaining goblet cells were counted and compared among groups.

\section{Terminal deoxynucleotidyl transferase-mediated dUTP nick end labeling (TUNEL) assay}

The formalin-fixed colonic sections were also subjected to TUNEL staining using a TUNEL assay kit (Roche Molecular Systems, MA, Switzerland). Nuclei were stained with $10 \mu \mathrm{g} / \mathrm{mL}$ Hoechst 33342 and images were acquired using a fluorescent microscope (Carl Zeiss AG, Jena, Germany). The number of apoptotic cells was counted as we described earlier [62].

\section{Transmission electron microscopy (TEM) analysis}

A $5 \mathrm{~mm}$ segment of fresh colon tissues were flushed with PBS and fixed in $2.5 \%$ glutaraldehyde at $4^{\circ} \mathrm{C}$ for $4 \mathrm{~h}$. After rinsed in PBS, the tissue was further fixed in PBS containing $1 \%$ osmium tetroxide for $2 \mathrm{~h}$ at room temperature, rinsed in PBS, and dehydrated. The tissues were then embedded in Epon 812 overnight. Embedding was then performed in Epon 812 and curing was done in an oven at $60^{\circ} \mathrm{C}$ for $48 \mathrm{~h}$. Sections of $80 \mathrm{~nm}$ thickness were cut on an ultramicrotome (RMC MTX) using a diamond knife. The sections were deposited on single-hole grids coated with Formvar and carbon and double-stained in aqueous solutions of $8 \%$ uranyl acetate for $25 \mathrm{~min}$ at $60^{\circ} \mathrm{C}$ and lead citrate for $3 \mathrm{~min}$ at room temperature. Images were acquired by a transmission HT7700 electron microscope (Hitachi, Tokyo, Japan).

\section{Quantification of inflammatory cytokines and antioxidant indexes in the colon and plasma}

A portion of frozen colon samples were homogenized with RIPA lysis buffer (Solarbio, Beijing, China) to extract total proteins. The homogenate was centrifuged at $12,000 \times \mathrm{g}$ at $4^{\circ} \mathrm{C}$ for $15 \mathrm{~min}$. The protein contents were quantified using a bicinchoninic acid (BCA) protein assay kit (Solarbio, Beijing, China) according to the manufacturer's instructions. The concentrations of proinflammatory cytokines such as IL-6 and TNF-a in the colon tissue were measured by ELISA (R\&D Systems, Minneapolis, MN, USA) according to the manufacturers' recommendations, while representative redox enzymes such as MPO, TSOD, CAT, T-AOC, GSH-px, and MDA were quantified using commercial kits (Nanjing Jiancheng Bioengineering Institute, Nanjing, China). The plasma concentrations of IL-1 $\beta$, IL-6, IL-8, TNF- $a$, T-AOC, CAT, and MDA in plasma were also measured similarly.

\section{RNA extraction and real-time quantitative PCR}

The remaining colonic tissues were subjected to total RNA isolation, cDNA synthesis, and RT-qPCR as we previously described [63]. Gene-specific primers were designed using Primer Premier 7.0 and their sequences are shown in Table S1. The qPCR reactions were performed on LightCycler ${ }^{\circledR} 96$ Real-Time PCR System (Roche Molecular Systems, MA, Switzerland) as follows: $95^{\circ} \mathrm{C}$ for 1 min, followed by 40 cycles of $95^{\circ} \mathrm{C}$ for $10 \mathrm{~s}$ and another $10 \mathrm{~s}$ at respective annealing temperature for each gene. Melting curve analysis was performed to verify the specificity of PCR reactions. The relative fold changes in the mRNA 
expression level of a gene were calculated using the $\Delta \Delta C$ t method [64] after normalization against $\beta$ actin.

\section{DNA extraction, 16S rRNA gene sequencing, and data analysis}

Total microbial genomic DNA of each fecal sample was extracted using QIAamp DNA Isolation Kit (Qiagen, Hilden, Germany). The concentration and integrity of DNA were assessed using Nanodrop (Thermo Fisher Scientific, USA) and 1.5\% agarose gel electrophoresis, respectively. Diluted DNA (1.0 $\mathrm{ng} / \mathrm{mL}$ ) was then used to amplify the V4 hypervariable region of the 16S rRNA gene with barcoded primers (515F, 5'-GTGCCAGCMGCCGCGGTAA-3', 806R, 5'-GGACTACHVGGGTWTCTAAT-3') and Phusion® High-Fidelity PCR Master Mix with GC Buffer (New England Biolabs, USA). PCR products were subjected to 2.0\% agarose gel electrophoresis, recovered, and purified using GeneJET Gel Extraction Kit (Thermo Fisher Scientific, USA) and then pooled into equal concentrations. An equal amount of DNA was used to prepare sequencing libraries with the lon Plus Fragment Library Kit (Thermo Fisher Scientific, USA) according to the manufacturer's recommendations. Library quality was assessed on Qubit 2.0 Fluorometer (Thermo Fisher Scientific, United States) and sequenced on the lon S5 ${ }^{\mathrm{TM}}$ XL platform (Thermo Fisher Scientific).

The low-quality reads were filtered with Cutadapt (V1.9.1) and chimeric sequences were removed after alignment with Gold database 1 using UCHIME Algorithm. The subsequent clean reads were clustered as operational taxonomic units (OTUs) using Uparse (version 7.0.1001) and annotated with the SILVA 16S rRNA gene database using MOTHUR program (version v.1.30.1). MUSCLE software (version 3.8.31) was applied to generate phylogenetic relationships of OTUs. Relative abundance of each OTU was then calculated. Alpha-diversity was calculated based on the profiles of OTU. Beta-diversity was estimated by calculating unweighted and weighted UniFrac distances and then visualized with principal coordinate analysis (PCOA). ANOSIM was performed to compare the similarity of bacterial communities among groups using the "vegan" package of R (version 3.3.1). Kyoto Encyclopedia of Genes and Genomes (KEGG) pathway enrichment analysis was performed to predict microbial functions. Linear discriminant analysis (LDA) effect size (LEfSe) was performed to identify the bacterial taxa differentially enriched in different bacterial communities.

\section{Statistical analysis}

All data were represented as means \pm SEM and analyzed using GraphPad Prism 8 program (GraphPad Software, San Diego, Canada). Data from more than two groups were compared using one-way ANOVA followed by Tukey's multiple comparison tests. $P \leq 0.05$ was considered statistically significant. Correlations between differentially enriched bacterial taxa and the concentrations of antioxidant enzymes, inflammatory cytokines or SCFAs were estimated by Spearman correlation analysis using the Pheatmap package in $\mathrm{R}$ (version 3.3.1).

\section{Declarations}


Acknowledgments

We thank all technicians in the experimental animal facility of China Agricultural University for providing daily care of mice. We also thank Shanghai Majorbio Bio-Pharm Technology Co. for their bioinformatic analysis of the data.

\section{Funding}

This work was supported by the National Natural Science Foundation of China $(31630074,31972596$, 31902170), the Beijing Municipal Natural Science Foundation (S170001), the National Key Research and Development Program of China (2016YFD0500506, 2018YDF0501002), the China Agriculture Research System (CARS-35), the 111 Project (B16044), and the Fundamental Research Funds for the Central Universities (2020TC067, 2020TC066).

\section{Availability of data and materials}

All data generated during this study are included in this manuscript and its supplementary files. Raw $16 \mathrm{~S}$ rRNA gene sequences for all samples used in this study have been deposited in the Sequence Read Archive (SRA) under Bioproject accession no. PRJNA613584.

\section{Ethics approval and consent to participate}

All animal experimental procedures were approved by the Institutional Animal Care and Use Committee of the China Agricultural University, Beijing, China, under protocol number AW24030202-1.

\section{Consent for publication}

Not applicable.

\section{Competing interests}

The authors have no conflicts of interest to declare.

\section{Authors' contributions}

The authors' contributions are as follows: JW, ZW, and SH designed the experiments. ZW and SH conducted the experiments. ZW, SH, JP, SW, and TL collected the samples and performed the analysis of samples. $\mathrm{ZW}, \mathrm{SH}$, and $\mathrm{NL}$ analyzed the data. $\mathrm{ZW}, \mathrm{SH}, \mathrm{DH}, \mathrm{GZ}$, and JW wrote the manuscript. All authors read and approved the final manuscript.

\section{References}

1. Xavier RJ, Podolsky DK: Unravelling the pathogenesis of inflammatory bowel disease. Nature 2007, 448(7152):427-434. 
2. Ng SC, Shi HY, Hamidi N, Underwood FE, Tang W, Benchimol El, Panaccione R, Ghosh S, Wu JCY, Chan FKL et al: Worldwide incidence and prevalence of inflammatory bowel disease in the 21st century: a systematic review of population-based studies. The Lancet 2017, 390(10114):2769-2778.

3. Franzosa EA, Sirota-Madi A, Avila-Pacheco J, Fornelos N, Haiser HJ, Reinker S, Vatanen T, Hall AB, Mallick $\mathrm{H}$, Mclver $\mathrm{LJ}$ et al: Gut microbiome structure and metabolic activity in inflammatory bowel disease. Nat Microbio/ 2019, 4(2):293-305.

4. Ramos GP, Papadakis KA: Mechanisms of Disease: Inflammatory Bowel Diseases. Mayo Clin Proc 2019, 94(1):155-165.

5. Nie JY, Zhao Q: Beverage consumption and risk of ulcerative colitis: Systematic review and metaanalysis of epidemiological studies. Medicine (Baltimore) 2017, 96(49):e9070.

6. Miranda PM, De Palma G, Serkis V, Lu J, Louis-Auguste MP, McCarville JL, Verdu EF, Collins SM, Bercik P: High salt diet exacerbates colitis in mice by decreasing Lactobacillus levels and butyrate production. Microbiome 2018, 6(1):57.

7. Burrello C, Garavaglia F, Cribiu FM, Ercoli G, Lopez G, Troisi J, Colucci A, Guglietta S, Carloni S, Guglielmetti $S$ et al: Therapeutic faecal microbiota transplantation controls intestinal inflammation through IL10 secretion by immune cells. Nat Commun 2018, 9(1):5184.

8. Zhu W, Winter MG, Byndloss MX, Spiga L, Duerkop BA, Hughes ER, Buttner L, de Lima Romao E, Behrendt CL, Lopez CA et al: Precision editing of the gut microbiota ameliorates colitis. Nature 2018, 553(7687):208-211.

9. Ng SC, Tang W, Leong RW, Chen M, Ko Y, Studd C, Niewiadomski O, Bell S, Kamm MA, de Silva HJ et al: Environmental risk factors in inflammatory bowel disease: a population-based case-control study in Asia-Pacific. Gut 2015, 64(7):1063-1071.

10. Piovani D, Danese S, Peyrin-Biroulet L, Nikolopoulos GK, Lytras T, Bonovas S: Environmental Risk Factors for Inflammatory Bowel Diseases: An Umbrella Review of Meta-analyses. Gastroenterology 2019, 157(3):647-659 e644.

11. Liu YC, Li XY, Shen L: Modulation effect of tea consumption on gut microbiota. App/ Microbiol Biotechnol 2020, 104(3):981-987.

12. Zhang S, Zhao Y, Ohland C, Jobin C, Sang S: Microbiota facilitates the formation of the aminated metabolite of green tea polyphenol (-)-epigallocatechin-3-gallate which trap deleterious reactive endogenous metabolites. Free Radic Biol Med 2019, 131:332-344.

13. Larabi A, Barnich N, Nguyen HTT: New insights into the interplay between autophagy, gut microbiota and inflammatory responses in IBD. Autophagy 2020, 16(1):38-51.

14. Aviello G, Singh AK, O'Neill S, Conroy E, Gallagher W, D'Agostino G, Walker AW, Bourke B, Scholz D, Knaus UG: Colitis susceptibility in mice with reactive oxygen species deficiency is mediated by mucus barrier and immune defense defects. Mucosal Immuno/ 2019, 12(6):1316-1326.

15. Lee Y, Sugihara K, Gillilland MG, Jon S, Kamada N, Moon JJ: Hyaluronic acid-bilirubin nanomedicine for targeted modulation of dysregulated intestinal barrier, microbiome and immune responses in colitis. Nature Materials 2019, 19(1):118-126. 
16. Cui H, Cai Y, Wang L, Jia B, Li J, Zhao S, Chu X, Lin J, Zhang X, Bian Y et al: Berberine Regulates Treg/Th17 Balance to Treat Ulcerative Colitis Through Modulating the Gut Microbiota in the Colon. Frontiers in Pharmacology 2018, 9.

17. Gan RY, Li HB, Sui ZQ, Corke H: Absorption, metabolism, anti-cancer effect and molecular targets of epigallocatechin gallate (EGCG): An updated review. Crit Rev Food Sci Nutr 2018, 58(6):924-941.

18. Chikara S, Nagaprashantha LD, Singhal J, Horne D, Awasthi S, Singhal SS: Oxidative stress and dietary phytochemicals: Role in cancer chemoprevention and treatment. Cancer Lett 2018, 413:122134.

19. Oz HS, Chen T, de Villiers WJ: Green Tea Polyphenols and Sulfasalazine have Parallel AntiInflammatory Properties in Colitis Models. Front Immunol 2013, 4:132.

20. Bruckner M, Westphal S, Domschke W, Kucharzik T, Lugering A: Green tea polyphenol epigallocatechin-3-gallate shows therapeutic antioxidative effects in a murine model of colitis. $J$ Crohns Colitis 2012, 6(2):226-235.

21. Chen D, Wan SB, Yang H, Yuan J, Chan TH, Dou QP: EGCG, green tea polyphenols and their synthetic analogs and prodrugs for human cancer prevention and treatment. Adv Clin Chem 2011, 53:155-177.

22. Microbiota fermentation-NLRP3 axis shapes the impact of dietary fibres on intestinal inflammation. Gut.

23. Vanilloid receptor-1 containing primary sensory neurones mediate dextran sulphate sodium induced colitis in rats.

24. Hansberry DR, Shah K, Agarwal P, Agarwal N: Fecal Myeloperoxidase as a Biomarker for Inflammatory Bowel Disease. Cureus 2017, 9(1):e1004.

25. Nguyen MM, Ahmann FR, Nagle RB, Hsu CH, Tangrea JA, Parnes HL, Sokoloff MH, Gretzer MB, Chow $\mathrm{HH}$ : Randomized, double-blind, placebo-controlled trial of polyphenon $\mathrm{E}$ in prostate cancer patients before prostatectomy: evaluation of potential chemopreventive activities. Cancer Prev Res (Phila) 2012, 5(2):290-298.

26. Graham DB, Xavier RJ: Pathway paradigms revealed from the genetics of inflammatory bowel disease. Nature 2020, 578(7796):527-539.

27. Ventham NT, Kennedy NA, Nimmo ER, Satsangi J: Beyond gene discovery in inflammatory bowel disease: the emerging role of epigenetics. Gastroenterology 2013, 145(2):293-308.

28. Du Y, Ding H, Vanarsa K, Soomro S, Baig S, Hicks J, Mohan C: Low dose Epigallocatechin Gallate Alleviates Experimental Colitis by Subduing Inflammatory Cells and Cytokines, and Improving Intestinal Permeability. Nutrients 2019, 11(8).

29. Molecular mechanisms underlying chemopreventive activities of anti-inflammatory phytochemicals: down-regulation of COX-2 and iNOS through suppression of NF-DB activation.

30. Du GJ, Zhang Z, Wen XD, Yu C, Calway T, Yuan CS, Wang CZ: Epigallocatechin Gallate (EGCG) is the most effective cancer chemopreventive polyphenol in green tea. Nutrients 2012, 4(11):1679-1691. 
31. Na HK, Surh YJ: Intracellular signaling network as a prime chemopreventive target of (-)epigallocatechin gallate. Mol Nutr Food Res 2006, 50(2):152-159.

32. Kale A, Gawande S, Kotwal S, Netke S, Roomi W, Ivanov V, Niedzwiecki A, Rath M: Studies on the effects of oral administration of nutrient mixture, quercetin and red onions on the bioavailability of epigallocatechin gallate from green tea extract. Phytother Res 2010, 24 Suppl 1:S48-55.

33. Xu XY, Zhao CN, Cao SY, Tang GY, Gan RY, Li HB: Effects and mechanisms of tea for the prevention and management of cancers: An updated review. Crit Rev Food Sci Nutr 2020, 60(10):1693-1705.

34. Littman DR, Pamer EG: Role of the commensal microbiota in normal and pathogenic host immune responses. Cell Host Microbe 2011, 10(4):311-323.

35. Dai Z, Coker OO, Nakatsu G, Wu WKK, Zhao L, Chen Z, Chan FKL, Kristiansen K, Sung JJY, Wong SH et al: Multi-cohort analysis of colorectal cancer metagenome identified altered bacteria across populations and universal bacterial markers. Microbiome 2018, 6(1):70.

36. Wang W, Zhai S, Xia Y, Wang H, Ruan D, Zhou T, Zhu Y, Zhang H, Zhang M, Ye H et al: Ochratoxin A induces liver inflammation: involvement of intestinal microbiota. Microbiome 2019, 7(1):151.

37. Epigallocatechin-3-gallate inhibits IL-6 synthesis and suppresses transsignaling by enhancing soluble gp130 production.

38. Xu Z, Wei C, Zhang RU, Yao J, Zhang D, Wang L: Epigallocatechin-3-gallate-induced inhibition of interleukin- 6 release and adjustment of the regulatory $T / T$ helper 17 cell balance in the treatment of colitis in mice. Exp Ther Med 2015, 10(6):2231-2238.

39. Bitzer ZT, Elias RJ, Vijay-Kumar M, Lambert JD: (-)-Epigallocatechin-3-gallate decreases colonic inflammation and permeability in a mouse model of colitis, but reduces macronutrient digestion and exacerbates weight loss. Mol Nutr Food Res 2016, 60(10):2267-2274.

40. Liu JB, Li JL, Zhuang K, Liu H, Wang X, Xiao QH, Li XD, Zhou RH, Zhou L, Ma TC et al: Epigallocatechin-3-gallate local preexposure application prevents SHIV rectal infection of macaques. Mucosal Immunol 2018, 11(4):1230-1238.

41. Aggarwal V, Tuli HS, Tania M, Srivastava S, Ritzer EE, Pandey A, Aggarwal D, Barwal TS, Jain A, Kaur $\mathrm{G}$ et al: Molecular mechanisms of action of epigallocatechin gallate in cancer: Recent trends and advancement. Semin Cancer Biol 2020.

42. Gil-Cardoso K, Gines I, Pinent M, Ardevol A, Blay M, Terra X: Effects of flavonoids on intestinal inflammation, barrier integrity and changes in gut microbiota during diet-induced obesity. Nutr Res Rev 2016, 29(2):234-248.

43. Suzuki T, Hara H: Role of flavonoids in intestinal tight junction regulation. J Nutr Biochem 2011, 22(5):401-408.

44. Wan ML, Ling KH, Wang MF, El-Nezami H: Green tea polyphenol epigallocatechin-3-gallate improves epithelial barrier function by inducing the production of antimicrobial peptide pBD-1 and pBD-2 in monolayers of porcine intestinal epithelial IPEC-J2 cells. Mol Nutr Food Res 2016, 60(5):1048-1058.

45. Green tea polyphenol (epigallocatechin3gallate) improves gut dysbiosis and serum bile acids dysregulation in highfat dietfed mice. 
46. Sheng LL, Jena PK, Liu HX, Hu Y, Nagar N, Bronner DN, Settles ML, Baumler AJ, Wan YJY: Obesity treatment by epigallocatechin-3-gallate-regulated bile acid signaling and its enriched Akkermansia muciniphila. Faseb J 2018, 32(12):6371-6384.

47. Xing L, Zhang H, Qi R, Tsao R, Mine Y: Recent Advances in the Understanding of the Health Benefits and Molecular Mechanisms Associated with Green Tea Polyphenols. J Agric Food Chem 2019, 67(4):1029-1043.

48. Li Y, Gao X, Lou Y: Interactions of tea polyphenols with intestinal microbiota and their implication for cellular signal conditioning mechanism. J Food Biochem 2019, 43(8):e12953.

49. Ushiroda C, Naito Y, Takagi T, Uchiyama K, Mizushima K, Higashimura Y, Yasukawa Z, Okubo T, Inoue R, Honda A et al: Green tea polyphenol (epigallocatechin-3-gallate) improves gut dysbiosis and serum bile acids dysregulation in high-fat diet-fed mice. J Clin Biochem Nutr 2019, 65(1):34-46.

50. Derrien M, Belzer C, de Vos WM: Akkermansia muciniphila and its role in regulating host functions. Microbial Pathogenesis 2017, 106:171-181.

51. Peterson SN, Bradley LM, Ronai ZA: The gut microbiome: an unexpected player in cancer immunity. Curr Opin Neurobiol 2020, 62:48-52.

52. Png CW, Linden SK, Gilshenan KS, Zoetendal EG, McSweeney CS, Sly LI, McGuckin MA, Florin TH: Mucolytic bacteria with increased prevalence in IBD mucosa augment in vitro utilization of mucin by other bacteria. Am J Gastroenterol 2010, 105(11):2420-2428.

53. Swidsinski A, Dorffel Y, Loening-Baucke V, Theissig F, Ruckert JC, Ismail M, Rau WA, Gaschler D, Weizenegger M, Kuhn S et al: Acute appendicitis is characterised by local invasion with Fusobacterium nucleatum/necrophorum. Gut 2011, 60(1):34-40.

54. Zhai R, Xue X, Zhang L, Yang X, Zhao L, Zhang C: Strain-Specific Anti-inflammatory Properties of Two Akkermansia muciniphila Strains on Chronic Colitis in Mice. Front Cell Infect Microbio/2019, 9:239.

55. von Schillde MA, Hormannsperger G, Weiher M, Alpert CA, Hahne H, Bauerl C, van Huynegem K, Steidler L, Hrncir T, Perez-Martinez G et al: Lactocepin secreted by Lactobacillus exerts antiinflammatory effects by selectively degrading proinflammatory chemokines. Cell Host Microbe 2012, 11(4):387-396.

56. Zagato E, Pozzi C, Bertocchi A, Schioppa T, Saccheri F, Guglietta S, Fosso B, Melocchi L, Nizzoli G, Troisi $\mathrm{J}$ et al: Endogenous murine microbiota member Faecalibaculum rodentium and its human homologue protect from intestinal tumour growth. Nat Microbiol 2020, 5(3):511-524.

57. Ivanov, II, Honda K: Intestinal commensal microbes as immune modulators. Cell Host Microbe 2012, 12(4):496-508.

58. Kayama H, Takeda K: Manipulation of epithelial integrity and mucosal immunity by host and microbiota-derived metabolites. Eur J Immunol 2020, 50(7):921-931.

59. Ruff WE, Greiling TM, Kriegel MA: Host-microbiota interactions in immune-mediated diseases. Nature Reviews Microbiology 2020. 
60. Peng Y, Yan Y, Wan P, Chen D, Ding Y, Ran L, Mi J, Lu L, Zhang Z, Li X et al: Gut microbiota modulation and anti-inflammatory properties of anthocyanins from the fruits of Lycium ruthenicum Murray in dextran sodium sulfate-induced colitis in mice. Free Radic Biol Med 2019, 136:96-108.

61. Stillie R, Stadnyk AW: Role of TNF receptors, TNFR1 and TNFR2, in dextran sodium sulfate-induced colitis. Inflammatory Bowel Diseases 2009, 15(10):1515-1525.

62. Ji Y, Dai Z, Sun S, Ma X, Yang Y, Tso P, Wu G, Wu Z: Hydroxyproline Attenuates Dextran Sulfate Sodium-Induced Colitis in Mice: Involvment of the NF-kappaB Signaling and Oxidative Stress. Mol Nutr Food Res 2018, 62(21):e1800494.

63. Huang S, Liu C, Li N, Wu Z, Li T, Han D, Li Z, Zhao J, Wang J: Membrane proteomic analysis reveals the intestinal development is deteriorated by intrauterine growth restriction in piglets. Funct Integr Genomics 2020, 20(2):277-291.

64. Bustin SA, Benes V, Garson JA, Hellemans J, Huggett J, Kubista M, Mueller R, Nolan T, Pfaffl MW, Shipley GL et al: The MIQE guidelines: minimum information for publication of quantitative real-time PCR experiments. Clin Chem 2009, 55(4):611-622.

\section{Description Of Supplementary Files}

Fig. S1. Rectal EGCG had a minimum effects on alleviating DSS-induced colitis. (A) Diagram illustrating the mouse model of colitis employed in this study. Rectal PBS and EGCG treatments were indicated. (B) Kinetics of DAl scores throughout the entire duration of the study. (C) Daily body weight changes throughout the entire duration of the study. Data were presented as Means \pm SEM ( $n=7$ per group). Statistical significance was determined using one-way ANOVA, followed by Turkey test. $* \star P \leq 0.01$, $* \star * P$ $\leq 0.001$ relative to Oral-CON group; \#\# $P \leq 0.01$, \#\#\# $P \leq 0.001$ relative to DSS + Oral-PBS group. (D) Macroscopic pictures of colons and (E) the lengths of colon from each group ( $n=7$ per group). (F) H\&E stained colon sections and $(G)$ histological scores of colons ( $n=6$ per group). Concentrations of four representative pro-inflammatory cytokines, IL-1 $1 \beta(\mathrm{H}), \mathrm{IL}-6(\mathrm{I})$, IL-8 (J), and TNF- $\alpha(\mathrm{K})$ in the plasma.

Concentration s of MPO (L), IL-6 (M), and TNF- $a(N)$ in the colon. Data were presented as Means \pm SEM ( $n$ = 7 per group). Statistical significance was determined using one-way ANOVA, followed by Turkey test. * $P \leq 0.05, * \star P \leq 0.01, * \star \star P \leq 0.001$.

Figure S2. Rectal EGCG played a minimum role in the anti-oxidative response in the plasma and colon. Concentrations of T-SOD (A), CAT (B), and MDA (C) in plasma from each group. Levels of T-AOC (D), T$\operatorname{SOD}(E), C A T(F), G S H-p x(G)$, and MDA $(H)$ in the colon. Data were presented as Means \pm SEM $(n=7$ per group). (I) Apoptosis rate in colonic sections. (J) Average of TUNEL-positive cells in colonic sections. Data were presented as Means \pm SEM ( $n=6$ per group). (K) Representative fluorescent pictures of TUNEL staining of colonic sections. Scale bars represent $50 \mu \mathrm{m}$. (L) Representative images of Alcian blue stained inner mucus layer of colonic sections. Scale bars represent $50 \mu \mathrm{m}$. (M) Representative images for the microstructure of colonic epithelia by TEM. Statistical significance was determined using one-way ANOVA, followed by Turkey test. * $P \leq 0.05, * \star P \leq 0.01$, *** $P \leq 0.001$. 
Fig. S3. Rectal EGCG altered the composition and function of the gut microbiota. (A) a-diversity upon oral therapy represented by the Sobs index. (B) PCoA plots upon rectal therapy assessed by Adonis. The relative abundance of fecal bacterial phyla (C), and genera (D) presented in $99.5 \%$ of the community upon rectal therapy. (E) Analysis of differences in the microbial taxa shown by LEfSe (LDA coupled with effect size measurements) upon rectal therapy. Only bacterial taxa reaching the LDA threshold of 2.0 are shown. (F) The 15 most significant KEGG pathways upon rectal therapy. Concentrations of fecal acetate $(\mathrm{G})$, propionate $(\mathrm{H})$, and butyrate $(\mathrm{I})$ upon rectal therapy. Data were presented as Means \pm SEM $(n=7$ per group). Statistical significance was determined using one-way ANOVA, followed by Turkey test. * $P \leq$ 0.05, , $P \leq 0.01$, , $* \star \quad P \leq 0.001$.

Fig. S4. Analysis of differences in the microbial taxa between the CON group and EGCG group was shown using LEfSe (LDA coupled with effect size measurements). Only bacterial taxa reaching the LDA threshold of 2.0 are shown.

Fig. S5. Bar plot showed the 15 most significant KEGG pathways between the CON group and EGCG group.

Fig. S6. Spearman Correlation between intestinal microbiota and anti-inflammatory or anti-oxidative parameters in healthy mice in response to prophylactic EGCG. The red color denotes a positive correlation, while green color denotes a negative correlation. The intensity of the color is proportional to the strength of Spearman correlation (R). * $P \leq 0.05,{ }^{\star *} P \leq 0.01$, ${ }^{\star \star *} P \leq 0.001$.

\section{Figures}


A

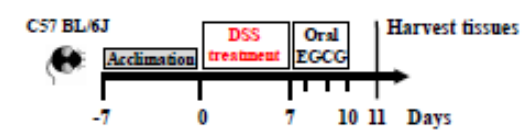

B

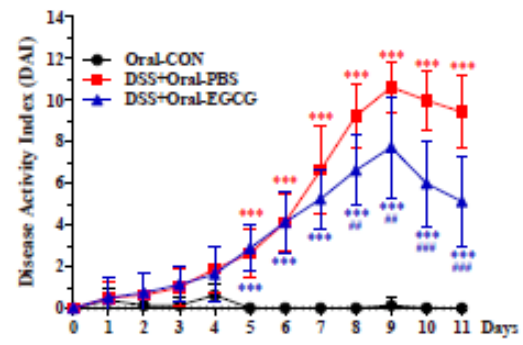

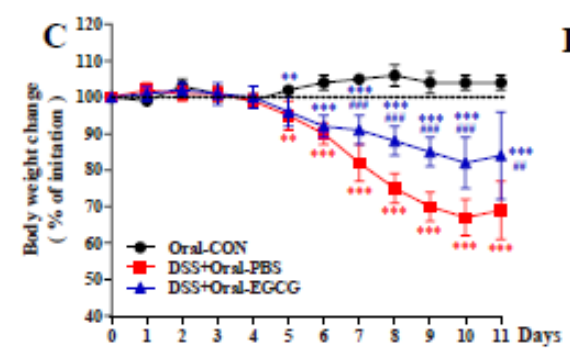

D
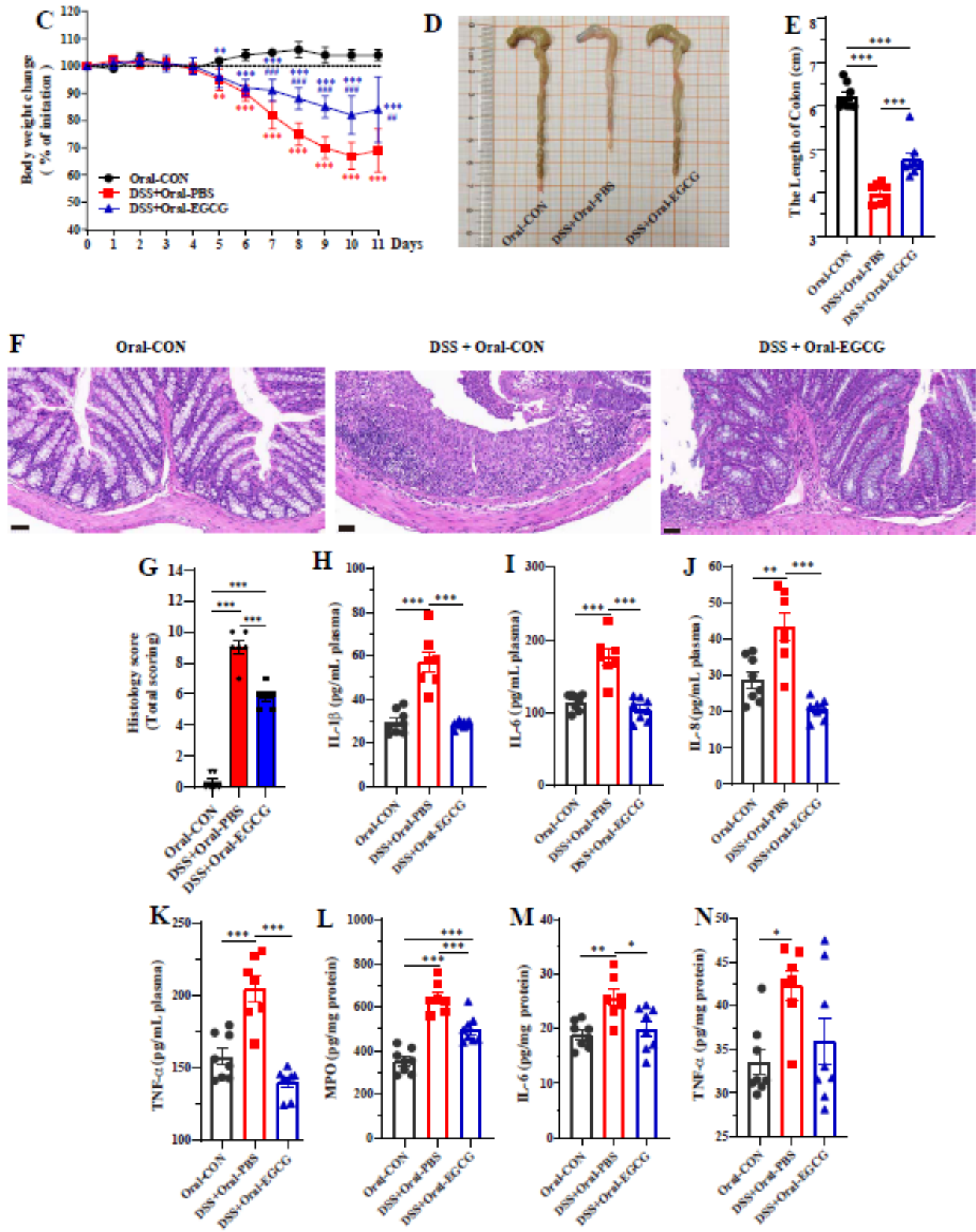

\section{Figure 1}

Oral administration of EGCG alleviated DSS-induced experimental colitis. (A) Diagram illustrating the mouse model of colitis employed in this study. Oral Phosphate buffer saline (PBS) and epigallocatechin3-gallate (EGCG) treatments are indicated. (B) Kinetics of daily disease activity index (DAI) scores throughout the entire duration of the study. (C) Daily body weight changes throughout the entire duration of the study. Data were presented as Means \pm SEM ( $n=7-8$ per group). Statistical significance was 
determined using one-way ANOVA, followed by Turkey test. ${ }^{\star *} P \leq 0.01$, ${ }^{\star \star *} P \leq 0.001$ relative to OralCON group; \#\# P $\leq 0.01$, \#\#\# $\mathrm{P} \leq 0.001$ relative to DSS + Oral-PBS group. (D) Macroscopic pictures of colons and ( $E$ ) the lengths of colon from each group ( $n=7-8$ per group). (F) H\&E stained colon sections and $(G)$ histological scores of colons ( $n=6$ per group). Concentrations of four representative proinflammatory cytokines, IL-1 $\beta(\mathrm{H})$, IL-6 (I), IL-8 (J), and TNF- $\alpha(\mathrm{K})$ in the plasma. Concentrations of myeloperoxidase (MPO) (L), IL-6 (M), and TNF- $a(N)$ in the colon. Data were presented as Means \pm SEM ( $n$ = 7-8 per group). Statistical significance was determined using one-way ANOVA, followed by Turkey test. * $P \leq 0.05, * \star P \leq 0.01, * \star \star P \leq 0.001$. 

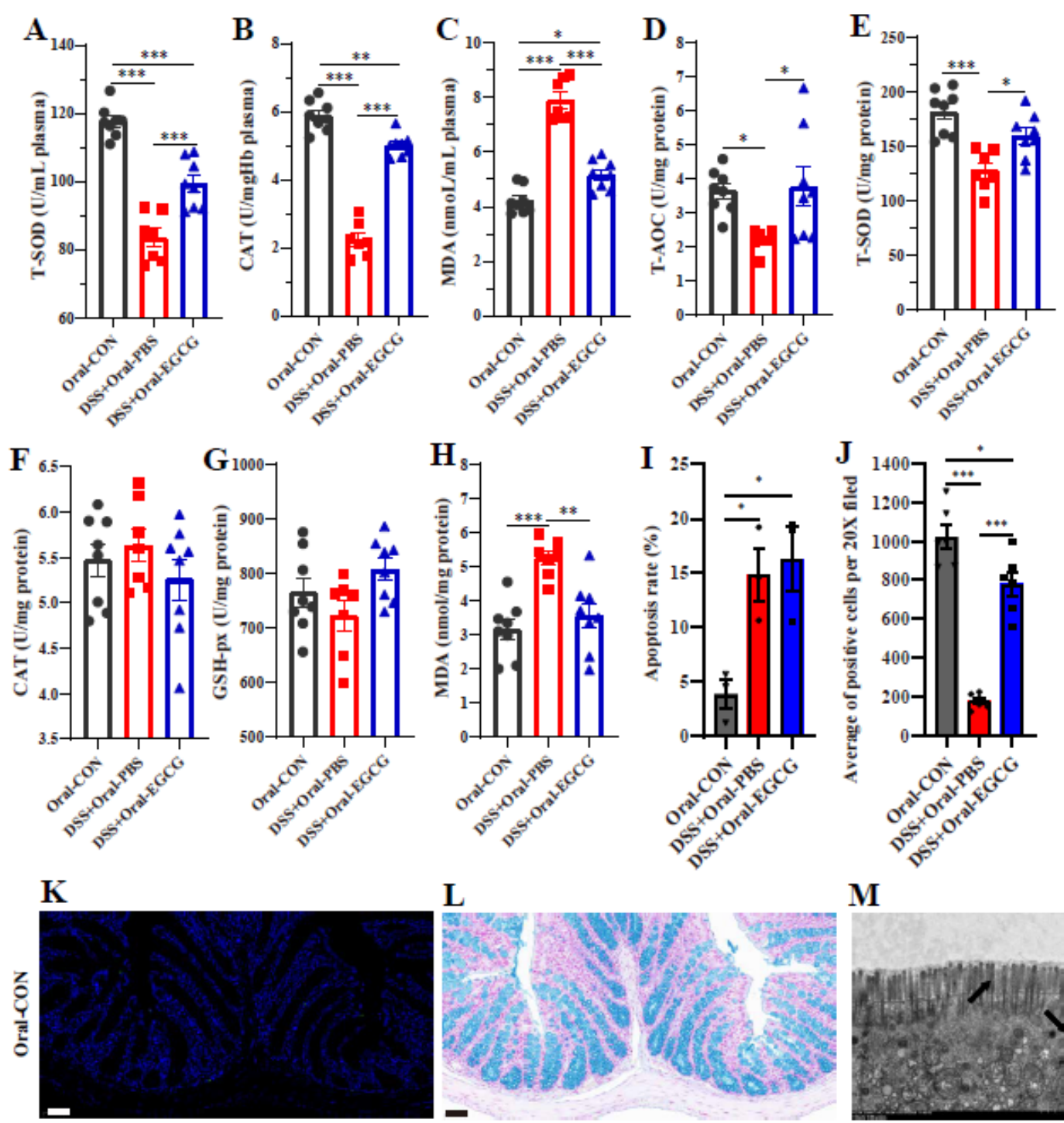

$\mathbf{L}$

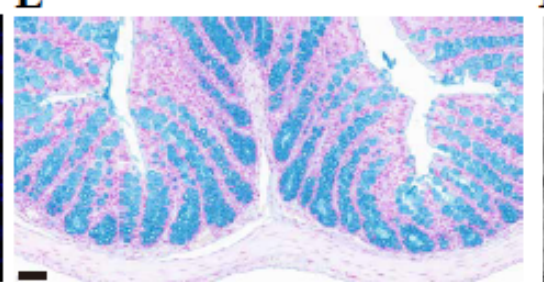

M
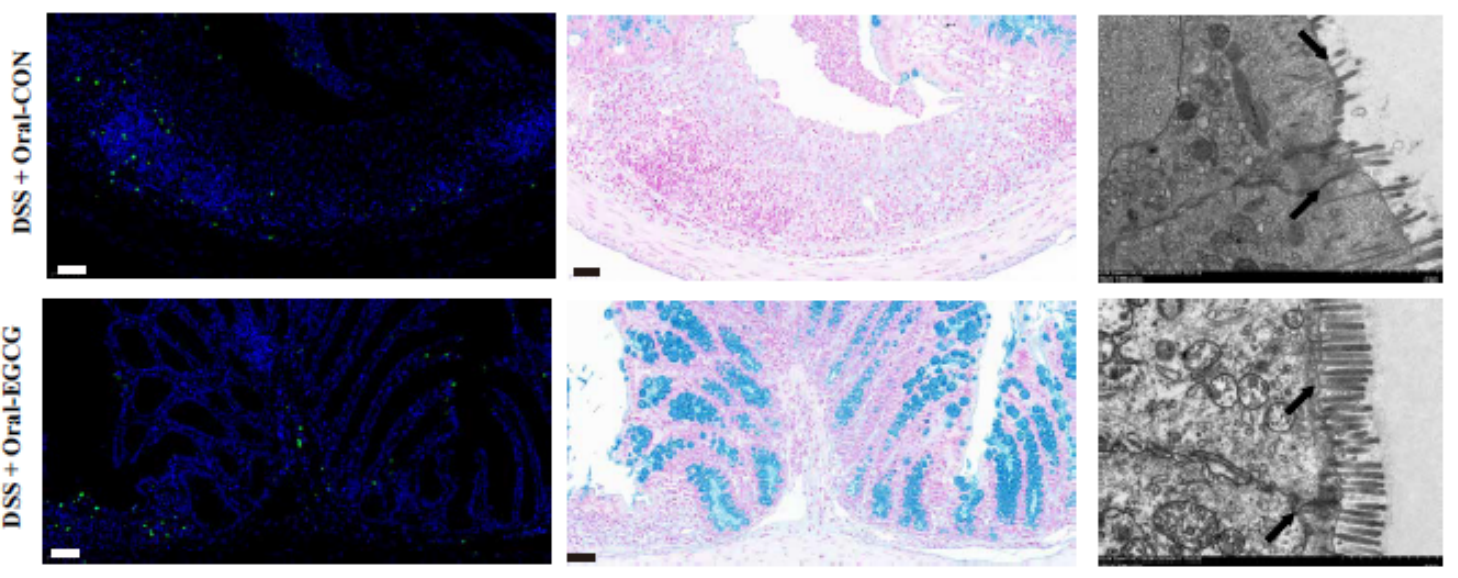

Figure 2

Oral EGCG attenuated oxidative stress in the plasma and colon. Concentrations of T-SOD (A), CAT (B), and MDA (C) in plasma from each group. (D) Level of T-AOC in the colon. Concentrations of T-SOD (E), CAT (F), GSH-px (G), and MDA (H) in the colon. Data are presented as Means \pm SEM ( $n=7-8$ per group). (I) Apoptosis rate in colonic sections. (J) Average of TUNEL-positive cell in colonic sections. Data were presented as Means \pm SEM ( $n=6$ per group). (K) Representative fluorescent pictures of TUNEL staining 
of colonic sections. Scale bars represent $50 \mu \mathrm{m}$. (L) Representative images of Alcian blue stained inner mucus layer of colonic sections. Scale bars represent $50 \mu \mathrm{m}$. (M) Representative images showing the microstructure of colonic epithelia by TEM. Statistical significance was determined using one-way ANOVA, followed by Turkey test. ${ }^{*} \mathrm{P} \leq 0.05$, $* * \mathrm{P} \leq 0.01$, *** $\mathrm{P} \leq 0.001$.
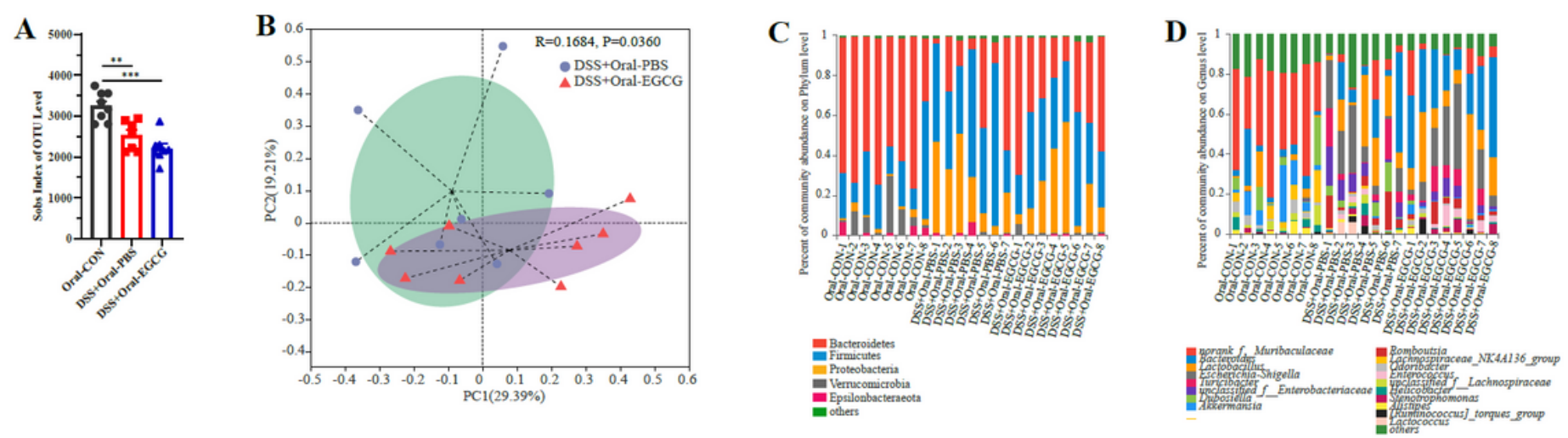

$\mathbf{E}$

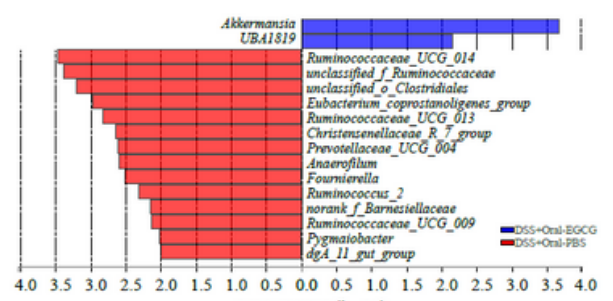
LDA SCORE( $(\log 10)$
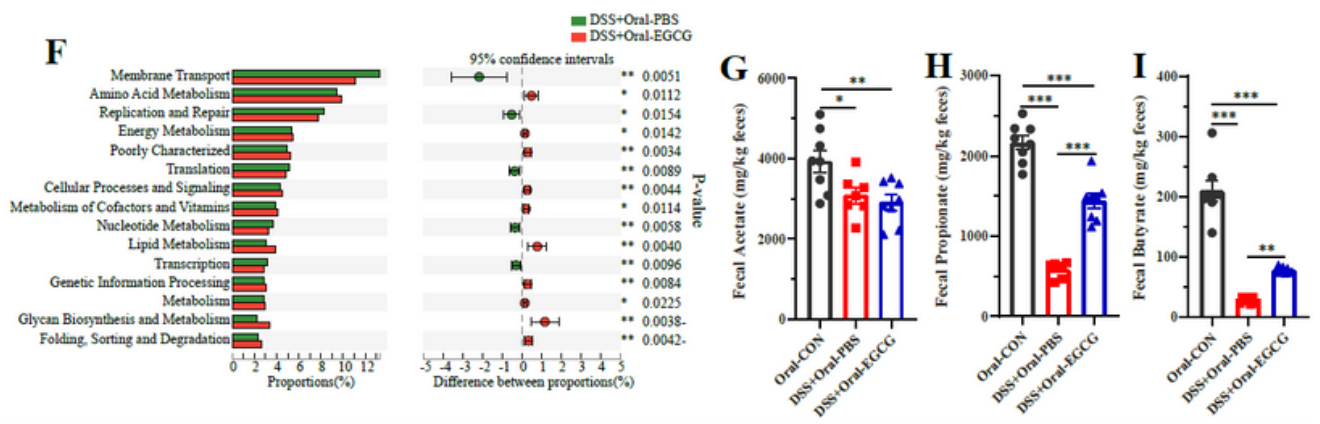

\section{Figure 3}

Oral EGCG regulated the composition and function of intestinal microbiota. (A) a-diversity upon oral therapy represented by the Sobs index. (B) Principal coordinate analysis (PCoA) plots upon oral therapy assessed by Adonis. The relative abundance of fecal bacterial phyla (C); and genera (D) presented in $99.5 \%$ of the community upon oral therapy. (E) Analysis of differences in the microbial taxa shown by LEfSe (linear discriminant analysis (LDA) coupled with effect size measurements) upon oral therapy. Only bacterial taxa reaching the LDA threshold of 2.0 are shown. (F) The 15 most significant KEGG pathways upon oral therapy. Concentrations of fecal acetate $(G)$, propionate $(H)$, and butyrate $(I)$ upon oral therapy. Data were presented as Means \pm SEM $(n=7-8$ per group). Statistical significance was determined using one-way ANOVA, followed by Turkey test. * $P \leq 0.05$, ${ }^{\star \star} P \leq 0.01$, ${ }^{\star \star \star} P \leq 0.001$. 


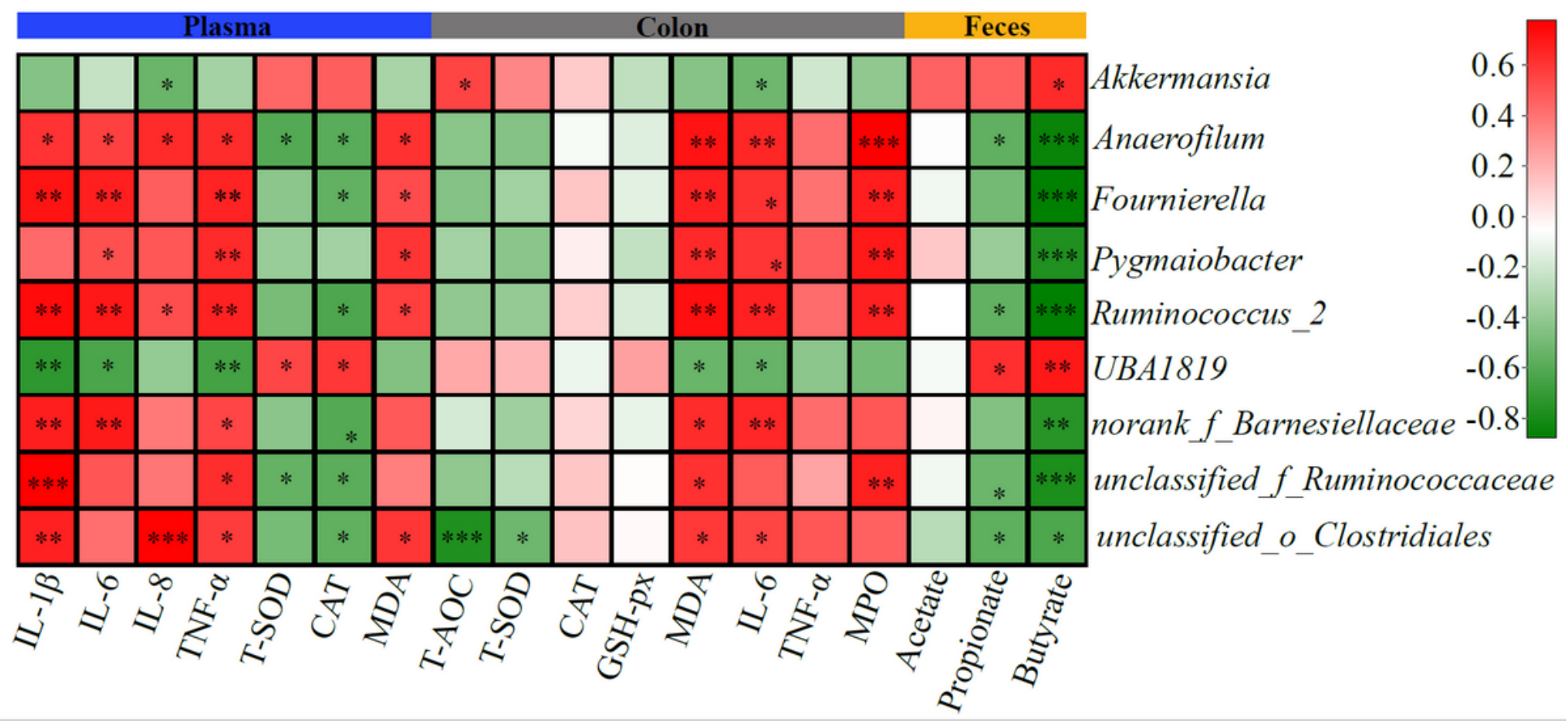

Figure 4

Spearman Correlation between intestinal microbiota and anti-inflammatory or anti-oxidative parameters in DSS-treated mice in response to oral EGCG. The red color denoted a positive correlation, while green color denoted a negative correlation. The intensity of the color was proportional to the strength of

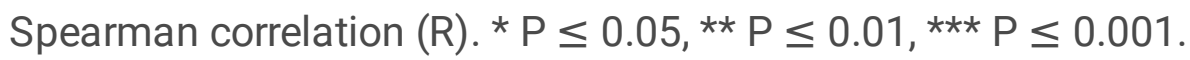


A

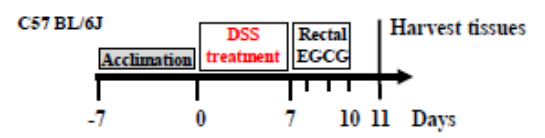

D
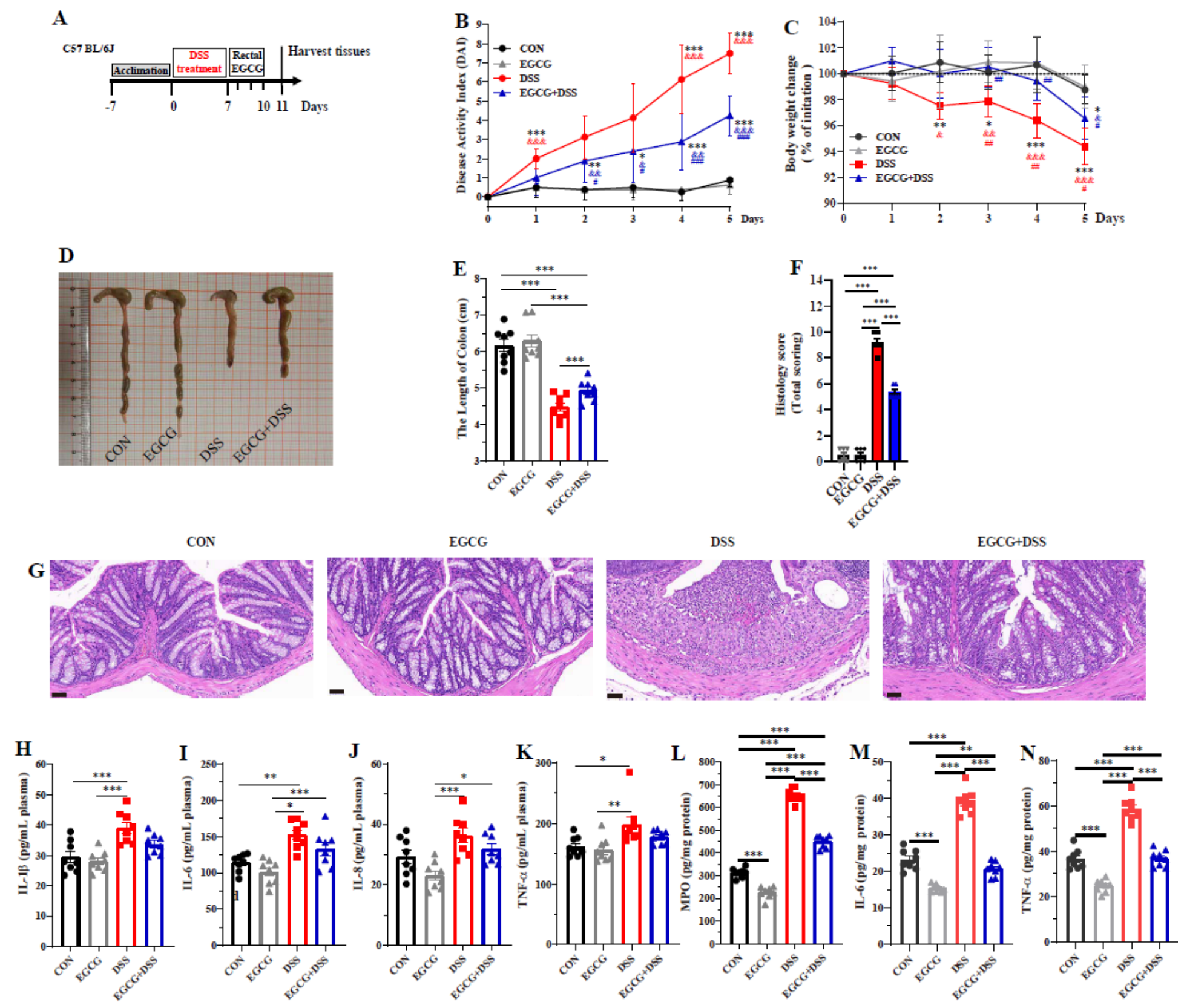

Figure 5

Prophylactic EGCG attenuated the symptoms of DSS-induced colitis. (A) Diagram illustrating the experimental design employed in this study. (B) Kinetics of DAI scores following DSS treatment. (C) Daily body weight changes following DSS treatment. Data were presented as Means \pm SEM ( $n=8$ per group). Statistical significance was determined using one-way ANOVA, followed by Turkey test. * $P \leq 0.05$, ** $P \leq$ 0.01 , $* \star * P \leq 0.001$ relative to $C O N$ group; $\& P \leq 0.05, \& \& P \leq 0.01, \& \& \& P \leq 0.001$ relative to EGCG group; $\# P \leq 0.05$, \#\# $\mathrm{P} \leq 0.01, \# \# \# \mathrm{P} \leq 0.001$ relative to DSS group. (D) Macroscopic pictures of colons and $(E)$ the lengths of colon from each group ( $n=8$ per group). ( $F)$ H\&E stained colon sections and $(G)$ histological scores of colons ( $n=6$ per group). Concentrations of four representative pro-inflammatory cytokines, IL-1 $\beta(H)$, IL-6 (I), IL-8 (J), and TNF- $a(K)$ in the plasma. Concentrations of MPO (L), IL-6 (M), and TNF- $a(N)$ in the colon. Data were presented as Means \pm SEM ( $n=8$ per group). Statistical 
significance was determined using one-way ANOVA, followed by Turkey test. * $P \leq 0.05$, $* * P \leq 0.01$, $* \star * P$ $\leq 0.001$.
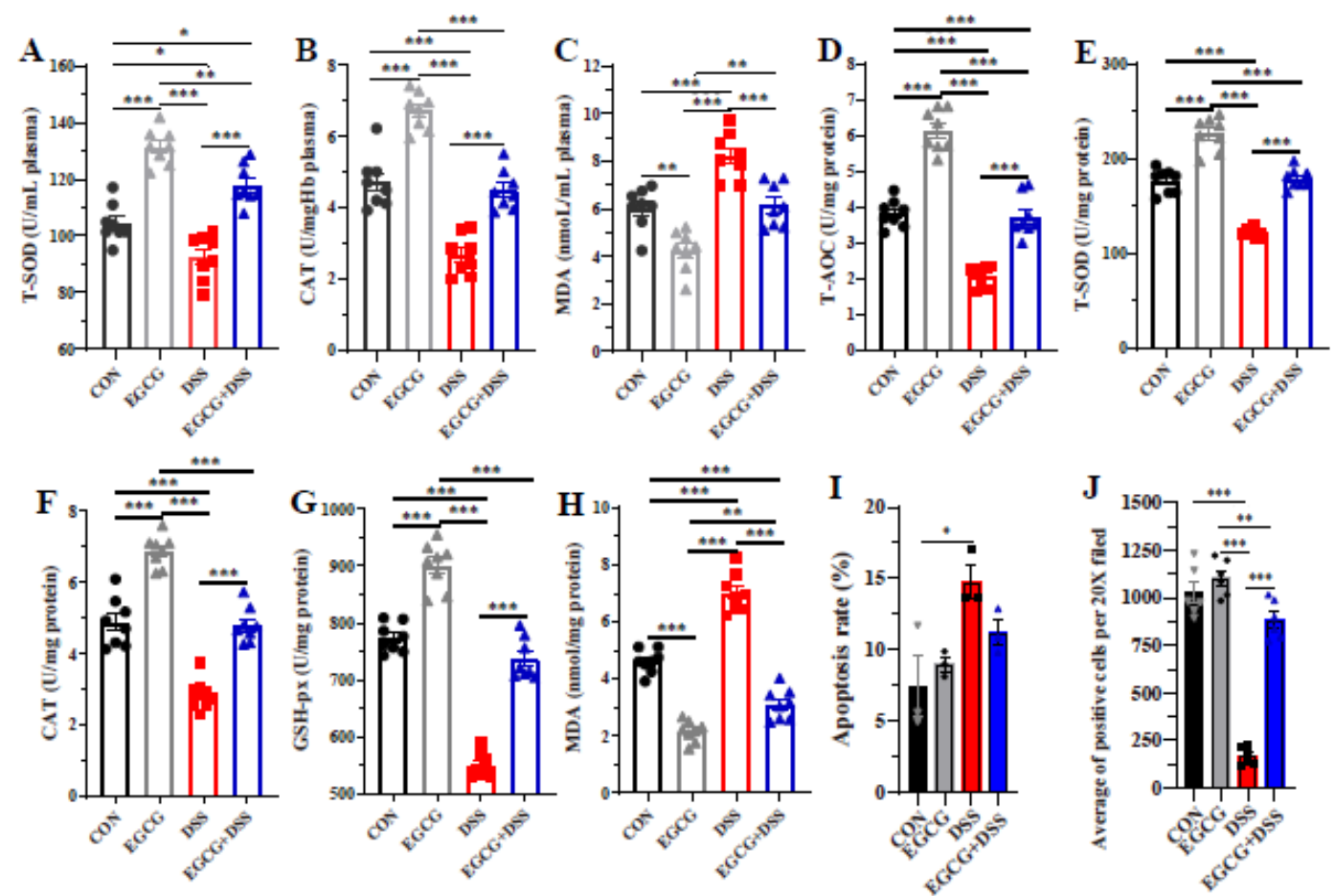

$\mathbf{K}$

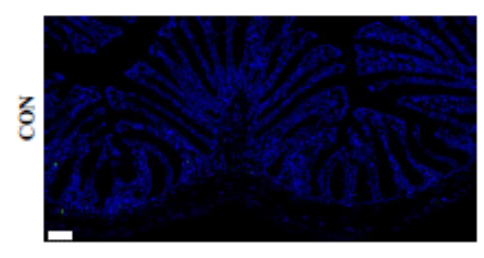

$\mathbf{L}$
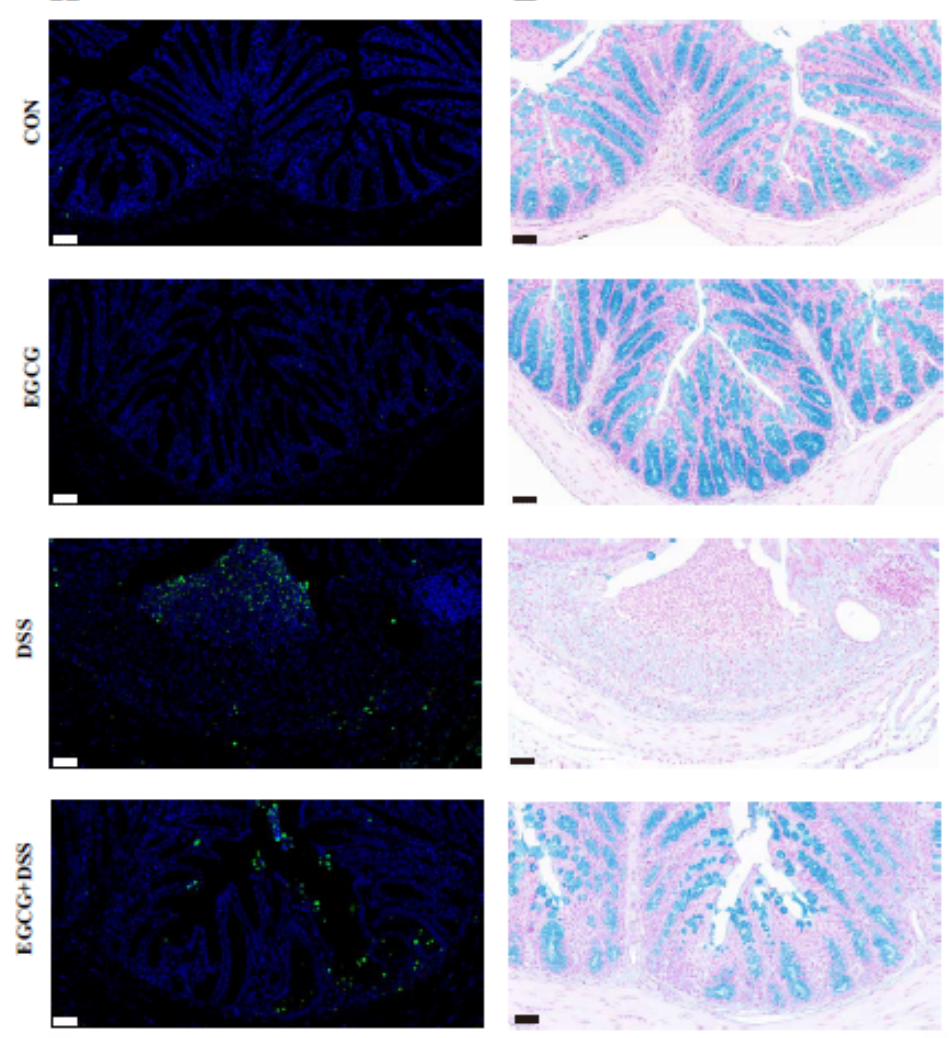

\section{M}
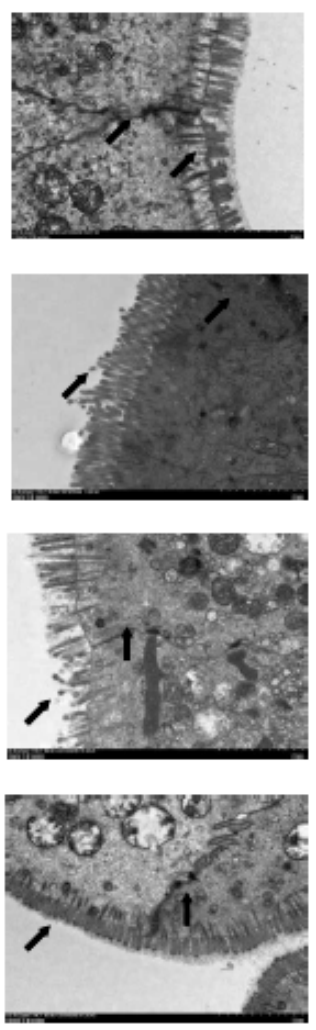

Figure 6

Prophylactic EGCG improved the anti-oxidative response in the plasma and colon. Concentrations of TSOD (A), CAT (B), and MDA (C) in the plasma. Levels of T-AOC (D), T-SOD (E), CAT (F), GSH-px (G), and $\operatorname{MDA}(\mathrm{H})$ in the colon. Data were presented as Means \pm SEM ( $\mathrm{n}=8$ per group). (I) Apoptosis rate in 
colonic sections. $(\mathrm{J})$ Average of TUNEL-positive cells in colonic sections. Data were presented as Means \pm SEM ( $n=6$ per group). (K) Representative fluorescent pictures of TUNEL staining of colonic sections. Scale bars represent $50 \mu \mathrm{m}$. (L) Representative images of Alcian blue stained inner mucus layer of colonic sections. Scale bars represent $50 \mu \mathrm{m}$. (M) Representative images for the microstructure of colonic epithelia by TEM. Statistical significance was determined using one-way ANOVA, followed by Turkey test. * $\mathrm{P} \leq 0.05, * * \mathrm{P} \leq 0.01, * * * \mathrm{P} \leq 0.001$

Claudin-1

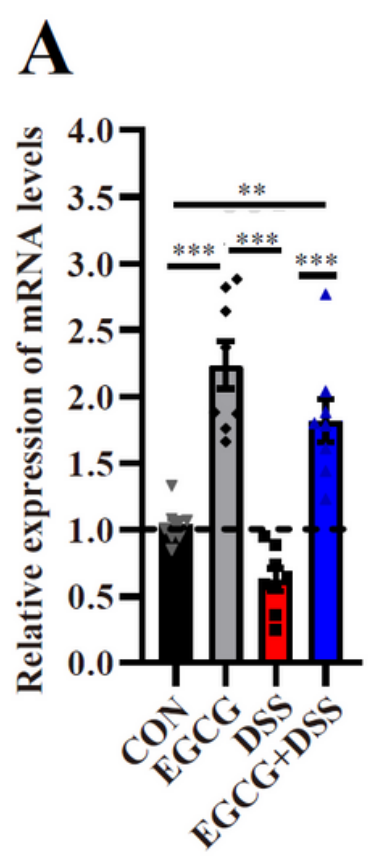

Ocuuludin

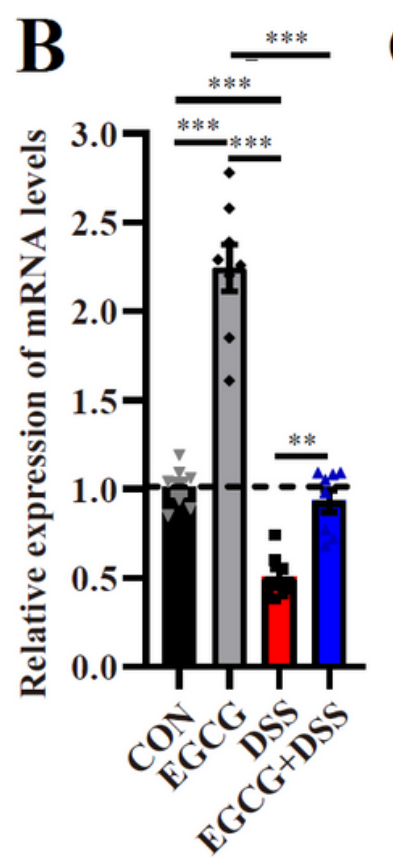

ZO-1

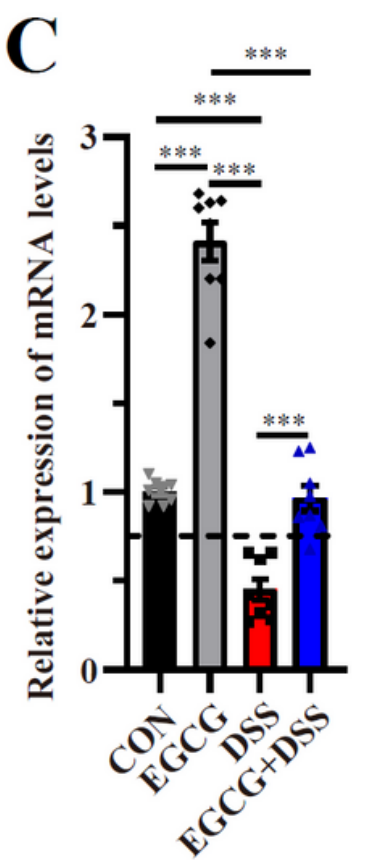

Mucin 1

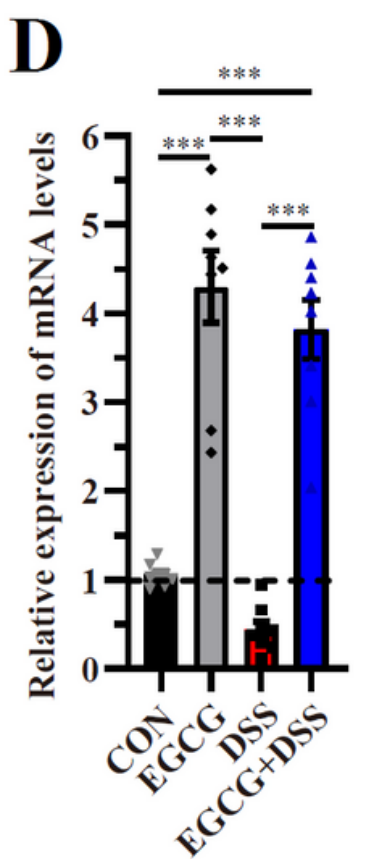

Mucin 2

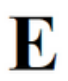

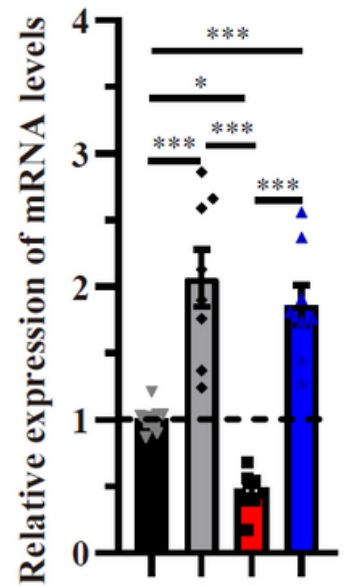

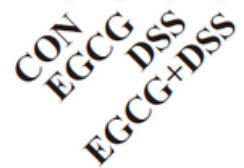

\section{Figure 7}

Prophylactic EGCG modulated the expression of the genes involved in intestinal barrier. Expression levels of Claudin-1(A), Occuludin (B), ZO-1 (C), Mucin 1 (D), and Mucin 2 (E) in the colon. Data are presented as Means \pm SEM ( $n=8$ per group). Statistical significance was determined using one-way ANOVA, followed by Turkey test. * $P \leq 0.05$, ** $P \leq 0.01$, *** $P \leq 0.001$.
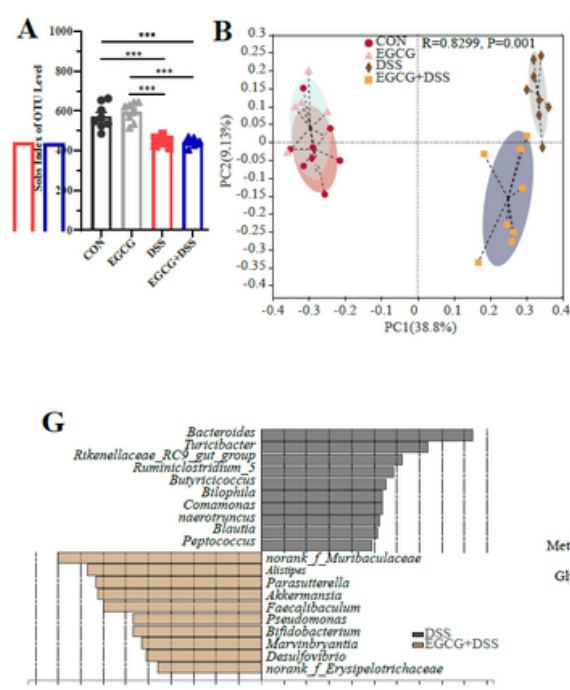

5.0454 .03 .53 .0252 .01 .51 .0050005101520253 .03 .54 .04 .55 .0
LDASCORE(10810)
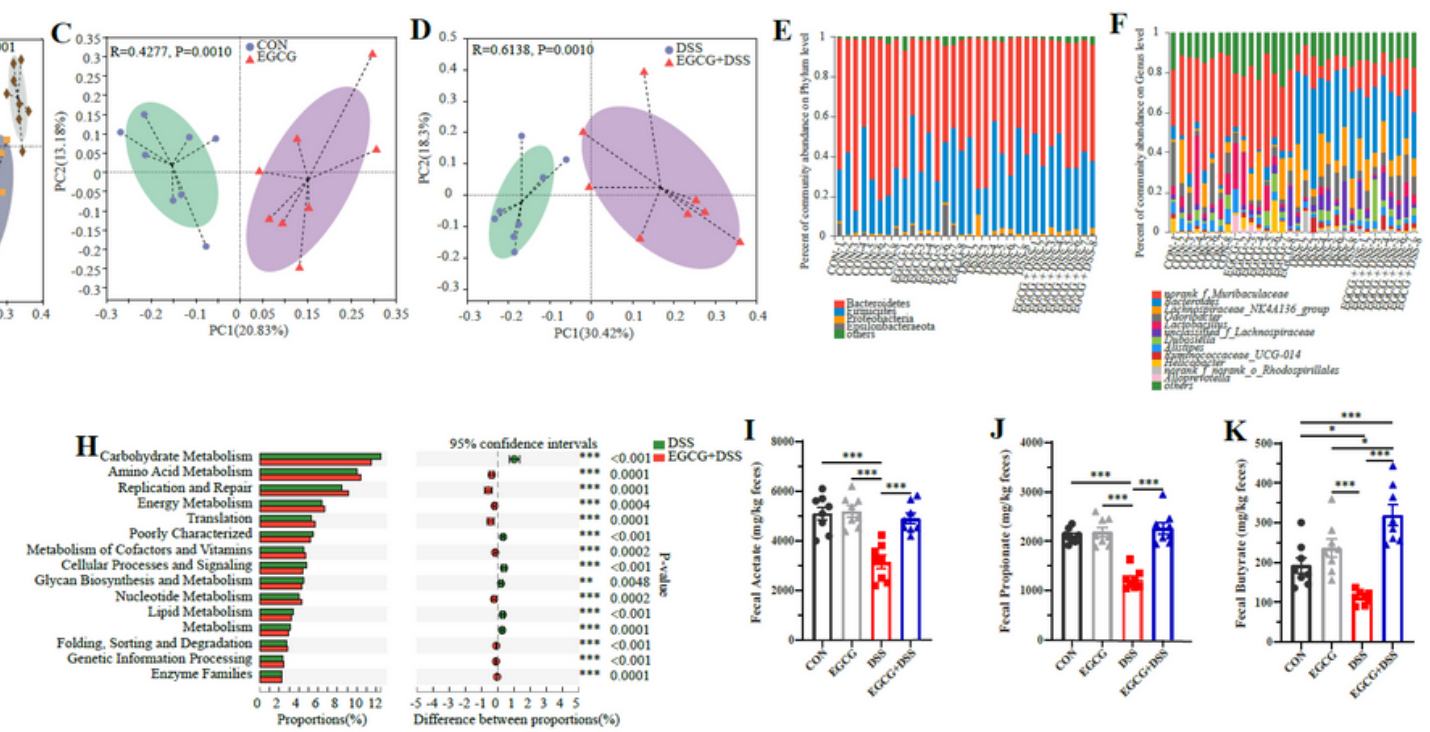


\section{Figure 8}

Prophylactic EGCG regulated the composition and function of intestinal microbiota. (A) a-diversity represented by the Sobs index on day 28. PCoA plots on day 28 assessed by Adonis among the four groups (B), between the two groups of healthy mice with or without receiving EGCG (C), and between the two groups of DSS-treated mice with or without receiving EGCG (D). The relative abundance of fecal bacterial phyla (E), and genera (F) presented in $99.5 \%$ of the community. (G) Analysis of differences in the microbial taxa shown by LEfSe (LDA coupled with effect size measurements) in DSS-treated mice with or without receiving EGCG on day 28. Only bacterial taxa reaching the LDA threshold of 2.0 are shown. $(H)$ The 15 most significant KEGG pathways between two groups with DSS on day 28. Concentrations of fecal acetate $(\mathrm{I})$, propionate $(\mathrm{J})$, and butyrate $(\mathrm{K})$ on day 28 . Data are presented as Means \pm SEM $(n=8$ per group). Statistical significance was determined using one-way ANOVA, followed by Turkey test. * $\mathrm{P} \leq$ $0.05, * \star P \leq 0.01, * \star \star P \leq 0.001$. 


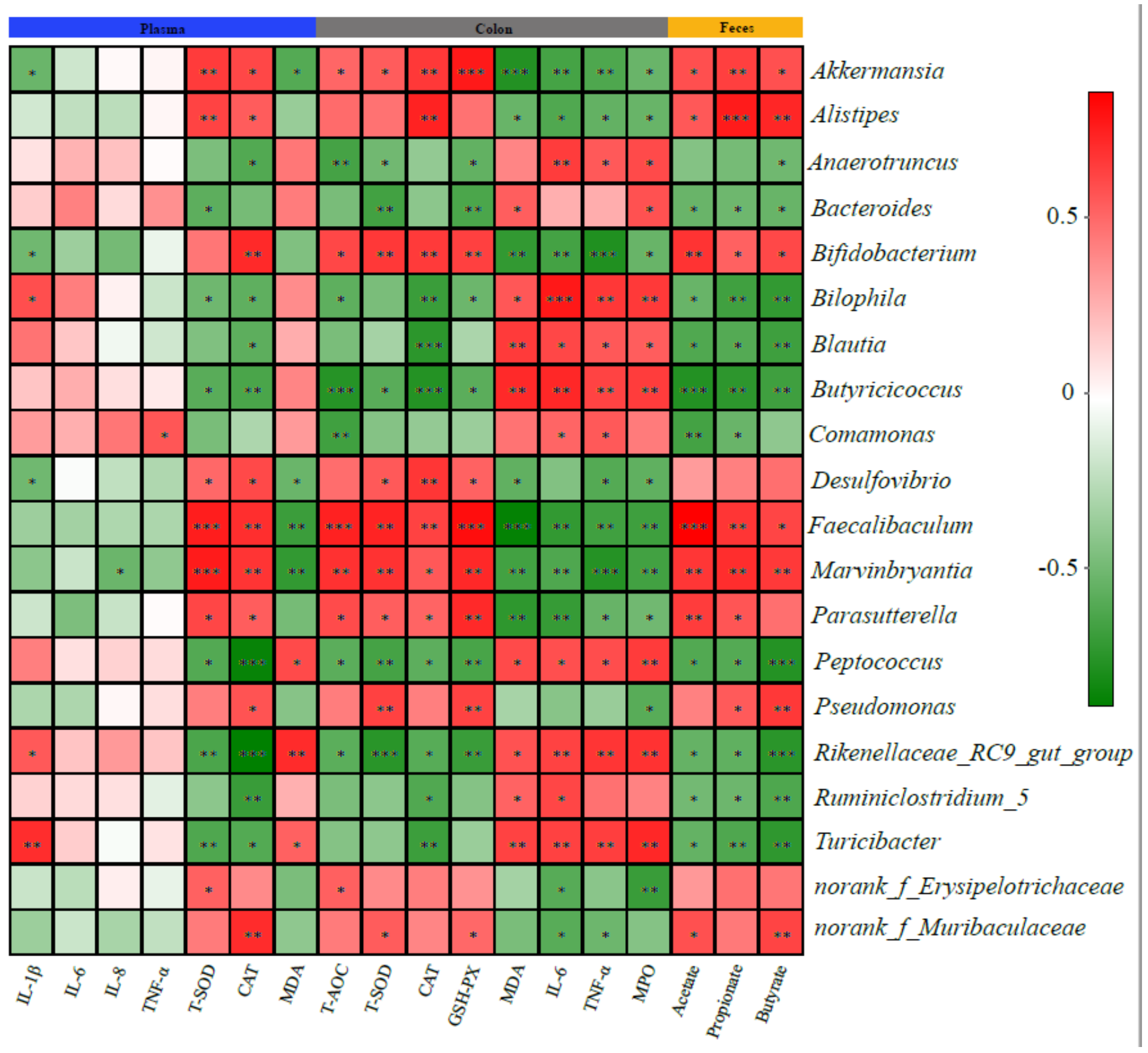

Figure 9

Spearman Correlation between intestinal microbiota and anti-inflammatory or anti-oxidative parameters in DSS-treated mice in response to prophylactic EGCG. The red color denotes a positive correlation, while green color denotes a negative correlation. The intensity of the color is proportional to the strength of Spearman correlation $(\mathrm{R}) .{ }^{*} \mathrm{P} \leq 0.05, * \star \mathrm{P} \leq 0.01, * \star * \mathrm{P} \leq 0.001$ 


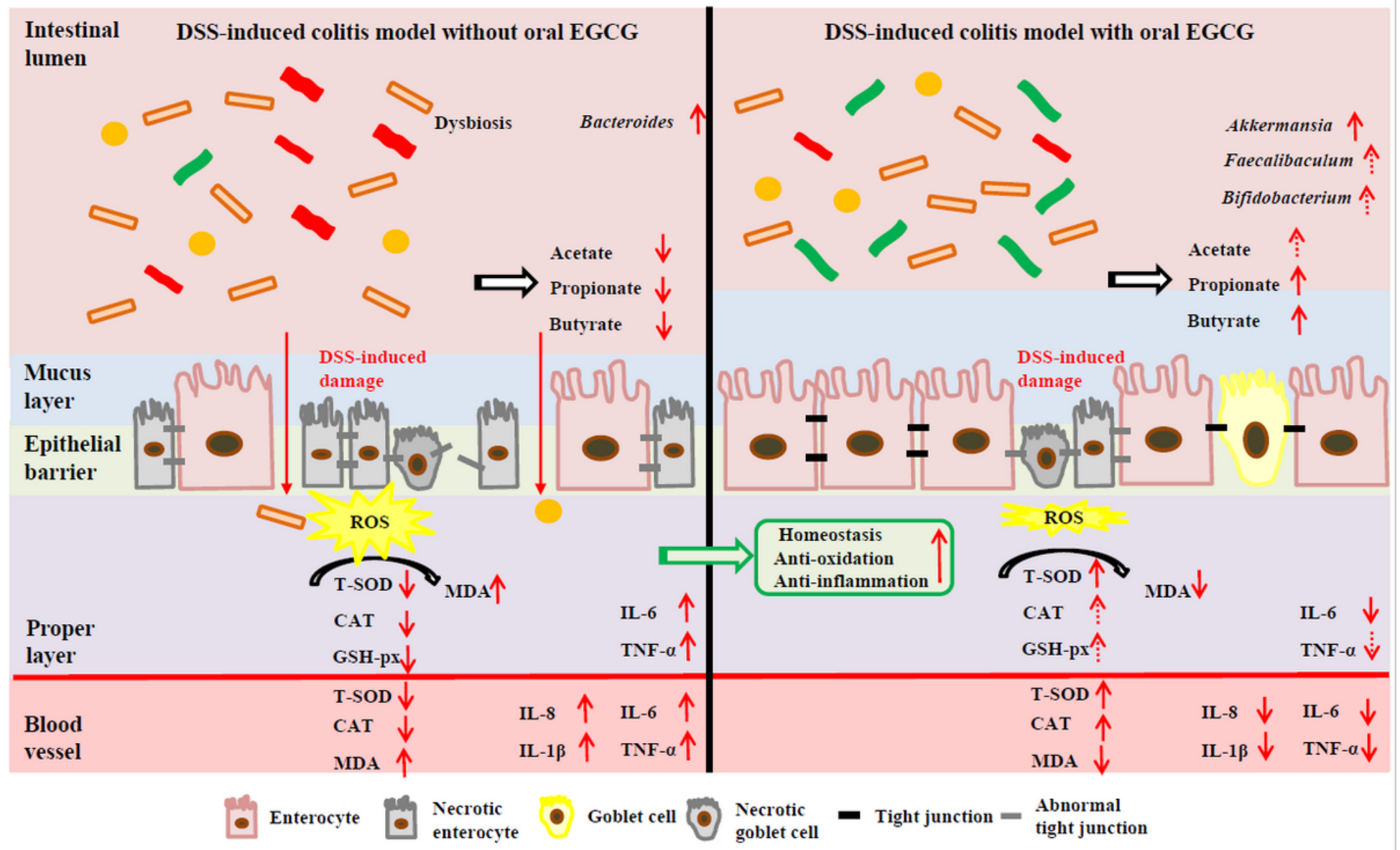

Figure 10

A schematic model showing the mechanism by which oral, but not rectal, delivery of EGCG alleviated DSS-induced colitis. Intestinal microbiota, oxidative stress, inflammation, and barrier integrity are all affected. Oral EGCG induced an alteration in the gut microbiota to enrich probiotic bacteria such as Akkermansia Faecalibaculum and Bifidobacterium, which subsequently led to an increased production of SCFAs such as butyrate, triggering a cascade of anti-oxidative, anti-inflammatory, and barrier-protective response. Ultimately, intestinal epithelial homeostasis is restored and colitis was attenuated.

\section{Supplementary Files}

This is a list of supplementary files associated with this preprint. Click to download.

- FigureS6.pdf

- Figures5.pdf

- FigureS4.pdf

- FigureS3.pdf

- Figures2.pdf

- Figures1.pdf 
- TableS1.docx

Page 33/33 